\title{
Volume-dependent pressure loading and its influence on the stability of structures
}

\author{
T. Rumpel ${ }^{1}$ and K. Schweizerhof ${ }^{2, *, \dagger}$ \\ ${ }^{1}$ Institut für Technische Mechanik, Universität Karlsruhe (TH), D-76128 Karlsruhe, Germany \\ ${ }^{2}$ Institut für Mechanik, Universität Karlsruhe (TH), D-76128 Karlsruhe, Germany
}

\begin{abstract}
SUMMARY
Deformation-dependent pressure loading on solid structures is created by the interaction of gas with the deformable surface of a structure. Such fairly simple load models are valid for static and quasistatic analyses and they are a very efficient tool to represent the influence of gas on the behaviour of structures. Completing previous studies on the deformation dependence of the loading with the assumption of infinite gas volumes, the current contribution is focusing on the influence of modifications of the size and shape of a finite volume containing the gas in particular on the stability of structures. The linearization of the corresponding virtual work expression necessary for a Newton-type solution leads to additional terms for the volume dependence. Investigating these terms the conservativeness of the problem can be proven by the symmetry of the linearized form. The discretization with finite elements leads to standard stiffness matrix forms plus the so-called load stiffness matrices and a rankone update for each enclosed volume part, if the loaded surface segments are identical with element surfaces. Some numerical examples show first the effectiveness of the approach and the necessity to take the corresponding terms in the variational expression and in the following linearization into account, and second the particular influence of this term on the stability of structures is shown with some specific examples. Copyright (c) 2002 John Wiley \& Sons, Ltd.
\end{abstract}

KEY WORDS: deformation-dependent pressure loading; volume dependence; large deformations; stability

\section{INTRODUCTION}

Pressure loading on structures usually originating from gas, from fluids or from wind action is mostly considered as static and uniform concerning deformation dependence within the considered processes. However, it is well known that this may be a rather dangerous view concerning the safety of structures even when only small deformations are considered. The most significant example is the stability load of cylinders under external pressure, where the stability load is reduced by a quarter even when considering small deformations only, if it is taken into account that the pressure remains normal to the surface [1]. For large deformations

\footnotetext{
${ }^{*}$ Correspondence to: K. Schweizerhof, Institut für Mechanik, Kaiserstrasse 12, Universität Karlsruhe (TH), D-76128 Karlsruhe, Germany

†E-mail: karl.schweizerhof@bau-verm.uni-karlsruhe.de
}

Copyright (C) 2002 John Wiley \& Sons, Ltd.

Received 21 September 2001

Revised 19 March 2002

Accepted 19 March 2002 
further effects appear such as the extension of pressure loaded surfaces and their motion within a loading field such as in water, see References [2,3] and the papers based on them [4-7]. Then it is rather important to consider the current position of the loaded part with respect to the water surface to get the correct loading. In addition for static considerations the mechanical effects of contents in silos can be represented via deformation dependent loading similar to unilateral contact, see [8].

If enclosed volumes are considered such as tires or airbags then the volume change within the loading process has a major effect on the local pressure. Similar problems occur if the structures show large deformations and contain fluids $[5,6]$ or gases $[9,10]$ and are otherwise loaded, such as water bags or tubes. The latter is of greater interest, if pneumatic structures are taken to achieve a local closure for structural parts as gas or pipe lines. In general volume effects are rather important for membrane-type structures as analysed by Oden et al. [11], and Gruttman et al. [12], where the focus is on the large deformation behaviour of hyperelastic membranes. For FE analyses of gas inflated structures and a good review we refer to Riches and Gosling $[13,14]$. A large number of other applications in practice show how important it is to have complete control over the pressure loading and its effects on the surrounding structures. Besides the recent study by Bonet et al. [15] with a strong focus on the hyperelastic large deformation behaviour of membranes, volume effects have been mainly taken into account in fluid structure analysis with complete FE models for fluid and structure parts and in airbag and hydroforming analysis. The latter applications are performed since several years with explicit FE programs as LS-DYNA [16] by the so-called control volumes considering the volume effect in a similar fashion as presented here. There only dynamic and accordingly quasistatic processes taking the inertia of the structures into account can be simulated; however, with very small time steps. Owing to the nature of the time integration algorithm - central differences - no information is available about stability, as no tangent matrices are set up.

Within the following our focus is on gas loading and on the corresponding derivations for static, respectively, implicit solution schemes to allow stability and large deformation FE analyses. Specifically, the conditions for conservativeness of the problems are also investigated.

\section{THE GOVERNING EQUATIONS}

The problem is prescribed by the external virtual work of a pressure load for the structure part and Poisson's law for the gas part considering a given initial volume.

\subsection{External virtual work of pressure loads resulting form gas acting on a given volume}

The deformation-dependent deformation of a pressurized structural part-containing a gas volume - can be described within the framework of virtual work via the external virtual work of a pressure load. The pressure $p$ is then dependent on the current volume $v(\mathbf{x})$ and thus on the current position $\mathbf{x}$ of all surface points of the structure. The external virtual work becomes:

$$
\delta \Pi_{\text {press }}=\int_{a} p \mathbf{n} \cdot \delta \mathbf{u} \mathrm{d} a
$$

with the normal $\mathbf{n}=\mathbf{x}_{, \xi} \times \mathbf{x}_{, \eta} /\left|\mathbf{x}_{, \xi} \times \mathbf{x}_{, \eta}\right|$, the surface element $\mathrm{d} a=\left|\mathbf{x}_{, \xi} \times \mathbf{x}_{, \eta}\right| \mathrm{d} \xi \mathrm{d} \eta$ and the pressure $p=p(v(\mathbf{x}))$. The surface vector $\mathbf{x}(\xi, \eta)$ depends on local co-ordinates $\xi, \eta$, 


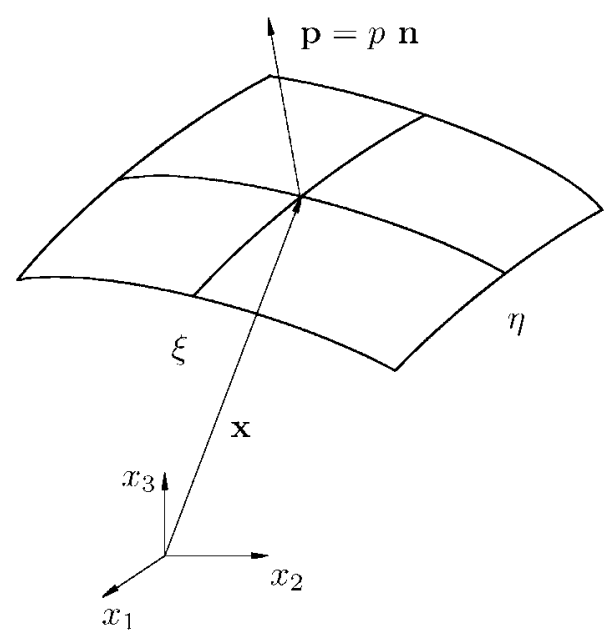

Figure 1. Surface under pressure loading.

see Figure 1.

$$
\delta \Pi_{\text {press }}=\int_{\eta} \int_{\xi} p\left(\mathbf{x}_{, \xi} \times \mathbf{x}_{, \eta}\right) \cdot \delta \mathbf{u} \mathrm{d} \xi \mathrm{d} \eta=\int_{\eta} \int_{\xi} p \mathbf{n}^{*} \cdot \delta \mathbf{u} \mathrm{d} \xi \mathrm{d} \eta
$$

Thus the pressure is acting perpendicular to the surface of the structure; the surface may be modified under loading in size $\mathrm{d} a$ and in direction represented by $\mathbf{n}$. In the general case the volume considered may consist out of one or more seperate but closed chambers filled with gas at different pressure. To simplify matters a non-normalized form $\mathbf{n}^{*}=\mathbf{x}_{, \xi} \times \mathbf{x}_{, \eta}$ of the normal vector $\mathbf{n}$ is introduced.

\subsection{Constitutive equation of the gas in a closed chamber}

The physical behaviour of the gas is specified by Poisson's law; $\kappa$ as isentropy constant, $P_{i}$, $V_{i}$ as initial pressure and volume for each closed chamber $i$ :

$$
p_{i} v_{i}^{\kappa}=P_{i} V_{i}^{\kappa}=\mathrm{const}
$$

For $\kappa=1$ the adiabatic change is simplified to Boyle's law, which is discussed in References [15]. The interesting aspect is the computation of the actual volume of the various enclosed chambers. Each volume $v_{i}$ is described by its corresponding surface and is computed via:

$$
v_{i}=\frac{1}{3} \int_{\eta} \int_{\xi} \mathbf{x} \cdot \mathbf{n}^{*} \mathrm{~d} \xi \mathrm{d} \eta
$$

\section{LINEARIZATION OF THE GOVERNING EQUATIONS}

If we consider a description of the structure with shell, volume or beam element surfaces and non-linear behaviour, the system of non-linear equations resulting from the variational expression must be linearized for the solution with a Newton-type scheme. Then the linearization at state $t$ is performed before the discretization with e.g., finite elements and within 


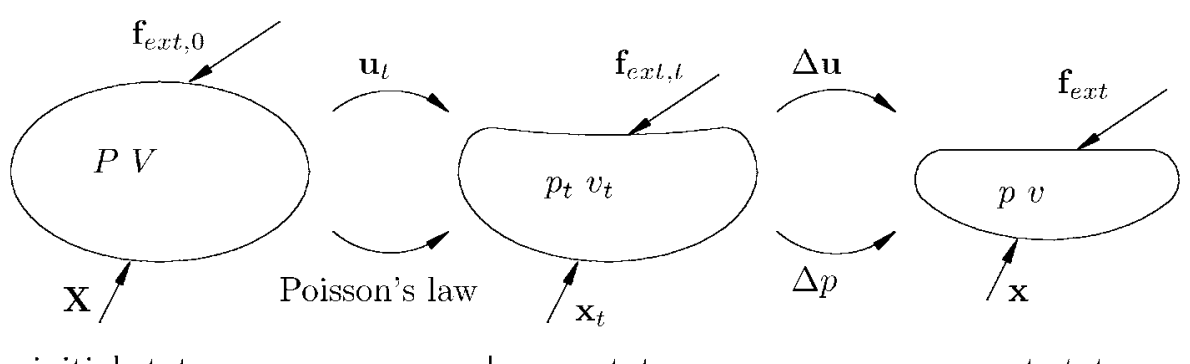

initial state

known state

current state

Figure 2. Newton scheme for volume-dependent pressure loading.

an incremental iterative procedure. The necessary quantities are $\mathbf{X}, V, P$ as initial position, volume and pressure, $\mathbf{x}_{t}=\mathbf{X}+\mathbf{u}_{t}, v_{t}=v_{t}\left(\mathbf{x}_{t}\right), p_{t}=p_{t}\left(P, V, v_{t}\right)$ as known position, volume and pressure at state $t$ and therefore $\mathbf{x}=\mathbf{x}_{t}+\Delta \mathbf{u}, v, p=p_{t}+\Delta p$ as position, volume and pressure at the current state. Within the chain rule the different contributions have to be linearized separately, see Figure 2.

\subsection{Linearization of the virtual work expression}

The expression for the external virtual work of one chamber $i$ including the linear part of the changes of the volume and the normal can be written as

$$
\begin{aligned}
& \delta \Pi_{i, \text { press }}^{\operatorname{lin}}=\delta \Pi_{\text {press }, t}+\delta \Pi_{\text {press }, t}^{\Delta p}+\delta \Pi_{\text {press }, t}^{\Delta \mathbf{n}} \\
& \delta \Pi_{i, \text { press }}^{\operatorname{lin}}=\int_{\eta} \int_{\xi}\left(p_{t} \mathbf{n}_{t}^{*} \cdot \delta \mathbf{u}+\Delta p \mathbf{n}_{t}^{*} \cdot \delta \mathbf{u}+p_{t} \Delta \mathbf{n}^{*} \cdot \delta \mathbf{u}\right) \mathrm{d} \xi \mathrm{d} \eta
\end{aligned}
$$

with

$$
\Delta \mathbf{n}^{*}=\Delta \mathbf{u}_{, \xi} \times \mathbf{x}_{t, \eta}-\Delta \mathbf{u}_{, \eta} \times \mathbf{x}_{t, \xi}
$$

for the non-normalized linearized normal vector.

\subsection{Linearization of Poisson's law}

As the gas law indicates that the product of its components remains constant, the change of the product is zero:

$$
\begin{aligned}
\Delta\left(p v^{\kappa}\right) & =0 \\
\Delta p v_{t}^{\kappa}+\Delta\left(v^{\kappa}\right) p_{t} & =0 \\
\text { whereby } \Delta\left(v^{\kappa}\right) & =\kappa \frac{v_{t}^{\kappa}}{v_{t}} \Delta v \\
\text { with } \Delta v & =\frac{1}{3} \int_{\eta} \int_{\xi}\left[\Delta \mathbf{u} \cdot \mathbf{n}_{t}^{*}+\mathbf{x}_{t} \cdot \Delta \mathbf{n}^{*}\right] \mathrm{d} \xi \mathrm{d} \eta \\
& \equiv \Delta v^{\Delta \mathbf{u}}+\Delta v^{\Delta \mathbf{n}}
\end{aligned}
$$


Finally the linearized expression for the adiabatic change needed in Equation (6) results in

$$
\Delta p+\frac{\kappa p_{t}}{v_{t}} \Delta v=0
$$

\section{CONSERVATIVENESS AND SYMMETRIC MATRICES}

It is known that the discussion of the conservativeness of the considered problem taking into account large deformations can be reduced to the discussion of the symmetry of the linearized expression concerning the displacements and the virtual displacements, see References $[1,17,18]$ for uniform pressure, [2,3] for non-uniform pressure fields and [4] for a summary based on Reference [2]. Within the following, we extend these considerations to chambers filled with gas, see also Reference $[9,10]$. If partial integration is used in a special fashion the linearized expression of the external virtual work and Poisson's law especially the enclosed volumes can be rewritten.

\subsection{Partial integration of the external virtual work-considering the change of the normal}

Our focus on the partial integration of the external virtual work is here restricted to the virtual work expression $\delta \Pi_{\text {press, },}^{\Delta \mathbf{n}}$, see Equations (5) and (6), which is a function of the linearized non-normalized normal vector $\Delta \mathbf{n}^{*}$. Introducing the covariant base vectors $\mathbf{g}_{\xi}=\mathbf{x}_{t, \xi}, \mathbf{g}_{\eta}=\mathbf{x}_{t, \eta}$, which denote the components of the boundary normal $\mathbf{n}_{t}^{s}$, see Figure 3, leads for the virtual work expression to:

$$
\begin{aligned}
\delta \Pi_{\text {press, }, t}^{\Delta \mathbf{n}} & =p_{t} \int_{\eta} \int_{\xi} \Delta \mathbf{n}^{*} \cdot \delta \mathbf{u} \mathrm{d} \xi \mathrm{d} \eta \\
& =p_{t} \int_{\eta} \int_{\xi}\left(\mathbf{g}_{\eta} \times \delta \mathbf{u}\right) \cdot \Delta \mathbf{u}_{, \xi}-\left(\mathbf{g}_{\xi} \times \delta \mathbf{u}\right) \cdot \Delta \mathbf{u}_{, \eta} \mathrm{d} \xi \mathrm{d} \eta
\end{aligned}
$$

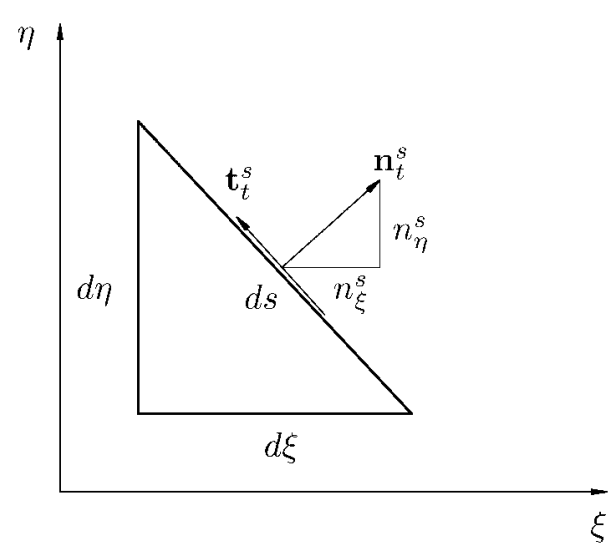

Figure 3. Configuration of the normal vector $\mathbf{n}_{t}^{s}$ and the tangent vector $\mathbf{t}_{t}^{s}$ at the boundary $s$. 


$$
\begin{aligned}
= & p_{t} \int_{s}\left(n_{\xi}^{s} \mathbf{g}_{\eta} \times \delta \mathbf{u}-n_{\eta}^{s} \mathbf{g}_{\xi} \times \delta \mathbf{u}\right) \cdot \Delta \mathbf{u} \mathrm{d} s \\
& -p_{t} \int_{\eta} \int_{\xi}\left[\frac{\partial}{\partial \xi}\left(\mathbf{g}_{\eta} \times \delta \mathbf{u}\right)-\frac{\partial}{\partial \eta}\left(\mathbf{g}_{\xi} \times \delta \mathbf{u}\right)\right] \cdot \Delta \mathbf{u} \mathrm{d} \xi \mathrm{d} \eta
\end{aligned}
$$

Assuming for illustration purpose a cartesian co-ordinates system at the boundary $s$ the normal vector $\mathbf{n}_{t}^{s}$ and the tangent vector $\mathbf{t}_{t}^{s}$ can be written as

$$
\left(\begin{array}{c}
\mathbf{n}_{t}^{s} \\
\mathbf{t}_{t}^{s}
\end{array}\right)=\left[\begin{array}{cc}
n_{\xi}^{s} & n_{\eta}^{s} \\
-n_{\eta}^{s} & n_{\xi}^{s}
\end{array}\right]\left(\begin{array}{l}
\mathbf{g}_{\xi} \\
\mathbf{g}_{\eta}
\end{array}\right)
$$

The relevant part of the external virtual work can then be written as

$$
\begin{aligned}
\delta \Pi_{\text {press }, t}^{\Delta \mathbf{n}}= & p_{t} \int_{s}\left(\Delta \mathbf{u} \times \mathbf{t}_{t}^{s}\right) \cdot \delta \mathbf{u} \mathrm{d} s \\
& -p_{t} \int_{\eta} \int_{\xi}\left(\mathbf{g}_{\eta} \times \delta \mathbf{u}_{, \xi}-\mathbf{g}_{\xi} \times \delta \mathbf{u}_{, \eta}\right) \cdot \Delta \mathbf{u} \mathrm{d} \xi \mathrm{d} \eta
\end{aligned}
$$

As already shown in References [2,3,8-10] the boundary integral vanishes - the first term in Equation (18) - if one of the following four conditions is valid:

C1 No loading $p$ on complete boundary $s: p=\left.0\right|_{s}$. (Only valid for fluid loading!)

$\mathrm{C} 2$ (a) Only displacements parallel to the boundary $s$ are allowed: $\Delta \mathbf{u} \| \mathbf{t}_{t}^{s} \rightarrow \Delta \mathbf{u} \times \mathbf{t}_{t}^{s}=\mathbf{0}$. This is physically clear as $\mathbf{t}_{t}^{s}$ is perpendicular to $p \mathbf{n}^{*}$ and the virtual work expression vanishes.

(b) One boundary displacement component perpendicular to the tangent vector is fixed. This corrects the statements given in References $[2,3,8,9]$ and by others.

C3 There is no boundary, thus only completely closed chambers filled with gas or fluid are considered $[9,10]$.

C4 The boundary integral vanishes as a whole which is hard to interprete physically, if there are no distinct boundary conditions.

For further conversions see References $[2,3,8]$ contravariant base vectors $\mathbf{g}^{\xi}, \mathbf{g}^{\eta}$ are introduced:

$$
-\mathbf{g}_{\xi}=\mathbf{n}^{*} \times \mathbf{g}^{\eta}, \quad \mathbf{g}_{\eta}=\mathbf{n}^{*} \times \mathbf{g}^{\xi}, \quad \mathbf{n}^{*}=\mathbf{g}_{\xi} \times \mathbf{g}_{\eta}
$$

The relevant part of the external virtual work expression becomes:

$$
\begin{aligned}
\delta \Pi_{\text {press }, t}^{\Delta \mathbf{n}} & =-p_{t} \int_{\eta} \int_{\xi}\left(\Delta \mathbf{u} \times \mathbf{n}^{*} \times \mathbf{g}^{\xi}\right) \cdot \delta \mathbf{u}_{, \xi}-\left(\Delta \mathbf{u} \times \mathbf{g}^{\eta} \times \mathbf{n}^{*}\right) \cdot \delta \mathbf{u}_{, \eta} \mathrm{d} \xi \mathrm{d} \eta \\
& =-p_{t} \int_{\eta} \int_{\xi}\left(\mathbf{n}^{*} \otimes \mathbf{g}^{\xi}-\mathbf{g}^{\xi} \otimes \mathbf{n}^{*}\right) \Delta \mathbf{u} \cdot \delta \mathbf{u}_{, \xi}+\left(\mathbf{n}^{*} \otimes \mathbf{g}^{\eta}-\mathbf{g}^{\eta} \otimes \mathbf{n}^{*}\right) \Delta \mathbf{u} \cdot \delta \mathbf{u}_{, \eta} \mathrm{d} \xi \mathrm{d} \eta
\end{aligned}
$$


For simplicity skew symmetric tensors $\underline{\mathbf{W}}^{\xi}, \underline{\mathbf{W}}^{\eta}$ are introduced, see also [8].

$$
\begin{aligned}
\delta \Pi_{\text {press }, t}^{\Delta \mathbf{n}} & =-p_{t} \int_{\eta} \int_{\xi} \underline{\mathbf{W}}^{\xi} \Delta \mathbf{u} \cdot \delta \mathbf{u}_{, \xi}+\underline{\mathbf{W}}^{\eta} \Delta \mathbf{u} \cdot \delta \mathbf{u}_{, \eta} \mathrm{d} \xi \mathrm{d} \eta \\
& =p_{t} \int_{\eta} \int_{\xi} \underline{\mathbf{W}}^{\xi \mathrm{T}} \Delta \mathbf{u} \cdot \delta \mathbf{u}_{, \xi}+\underline{\mathbf{W}}^{\eta \mathrm{T}} \Delta \mathbf{u} \cdot \delta \mathbf{u}_{, \eta} \mathrm{d} \xi \mathrm{d} \eta
\end{aligned}
$$

Following the proposal given in References [2,3] the relevant part of the virtual work expression given in Equation (14) can also be expressed in a slightly different manner:

$$
\begin{aligned}
\delta \Pi_{\text {press }, t}^{\Delta \mathbf{n}} & =p_{t} \int_{\eta} \int_{\xi}\left(\Delta \mathbf{u}_{, \xi} \times \mathbf{n}^{*} \times \mathbf{g}^{\xi}-\Delta \mathbf{u}_{, \eta} \times \mathbf{g}^{\eta} \times \mathbf{n}^{*}\right) \cdot \delta \mathbf{u} \mathrm{d} \xi \mathrm{d} \eta \\
& =p_{t} \int_{\eta} \int_{\xi}\left[\left(\mathbf{n}^{*} \otimes \mathbf{g}^{\xi}-\mathbf{g}^{\xi} \otimes \mathbf{n}^{*}\right) \Delta \mathbf{u}_{, \xi}+\left(\mathbf{n}^{*} \otimes \mathbf{g}^{\eta}-\mathbf{g}^{\eta} \otimes \mathbf{n}^{*}\right) \Delta \mathbf{u}_{, \eta}\right] \cdot \delta \mathbf{u} \mathrm{d} \xi \mathrm{d} \eta \\
& =p_{t} \int_{\eta} \int_{\xi}\left[\underline{\mathbf{W}}^{\xi} \Delta \mathbf{u}_{, \xi}+\underline{\mathbf{W}}^{\eta} \Delta \mathbf{u}_{, \eta}\right] \cdot \delta \mathbf{u} \mathrm{d} \xi \mathrm{d} \eta
\end{aligned}
$$

As the virtual work given by Equations (23) and (26) is identical, the considered part of the virtual work expression can now be rewritten in a symmetric form

$$
\begin{aligned}
\delta \Pi_{\mathrm{press}, t}^{\Delta \mathbf{n}} & =\frac{1}{2} \text { ‘Equation (23)’ }+\frac{1}{2} \text { 'Equation (26)’ } \\
& =\frac{p_{t}}{2} \int_{\eta} \int_{\xi}\left(\begin{array}{c}
\delta \mathbf{u} \\
\delta \mathbf{u}_{, \xi} \\
\delta \mathbf{u}_{, \eta}
\end{array}\right) \cdot\left[\begin{array}{ccc}
0 & \underline{\mathbf{W}^{\xi}} & \underline{\mathbf{W}}^{\eta} \\
\underline{\mathbf{W}}^{\xi \mathrm{T}} & 0 & 0 \\
\underline{\mathbf{W}}^{\eta \mathrm{T}} & 0 & 0
\end{array}\right]\left(\begin{array}{c}
\Delta \mathbf{u} \\
\Delta \mathbf{u}_{, \xi} \\
\Delta \mathbf{u}_{, \eta}
\end{array}\right) \mathrm{d} \xi \mathrm{d} \eta
\end{aligned}
$$

\subsection{Partial integration of Poisson's law-considering the change of the normal}

In analogy to Section 4.1 the partial integration of the gas law, see Equations (11)-(13), is restricted to the change of the normal $\Delta \mathbf{n}^{*}$ in the volume computation, see also the transformations given in Equations (14)-(18).

$$
\begin{aligned}
\Delta v^{\Delta \mathbf{n}} & =\frac{1}{3} \int_{\eta} \int_{\xi} \mathbf{x}_{t} \cdot \Delta \mathbf{n}^{*} \mathrm{~d} \xi \mathrm{d} \eta \\
& =\frac{1}{3} \int_{s}\left(\Delta \mathbf{u} \times \mathbf{t}_{t}^{s}\right) \cdot \mathbf{x}_{t} \mathrm{~d} s+\frac{2}{3} \int_{\eta} \int_{\xi} \mathbf{n}_{t}^{*} \cdot \Delta \mathbf{u} \mathrm{d} \xi \mathrm{d} \eta
\end{aligned}
$$

The first term in Equation (29) vanishes per definition for closed volumes and those with sliding boundary conditions which also lead to closed volumes. The adiabatic change of enclosed volumes can be rewritten as

$$
\Delta p+\kappa \frac{p_{t}}{v_{t}} \int_{\eta} \int_{\xi} \mathbf{n}_{t}^{*} \cdot \Delta \mathbf{u} \mathrm{d} \xi \mathrm{d} \eta=0
$$


Introducing Equation (30) into the external virtual work expression leads for the relevant part $\delta \Pi_{\text {press, } t}^{\Delta p}$, see Equation (5) and (6), to

$$
\delta \Pi_{\text {press }, t}^{\Delta p}=-\frac{\kappa p_{t}}{v_{t}} \int_{\eta} \int_{\xi} \mathbf{n}_{t}^{*} \cdot \Delta \mathbf{u} \mathrm{d} \xi \mathrm{d} \eta \int_{\eta} \int_{\xi} \mathbf{n}_{t}^{*} \cdot \delta \mathbf{u} \mathrm{d} \xi \mathrm{d} \eta
$$

\subsection{Symmetric tangent matrices and mixed formulation}

Considering the complete linearization, we sum up the separately considered parts in Sections 4.1 and 4.2.

$$
\begin{gathered}
\delta \Pi_{i, \text { press }}^{\operatorname{lin}}=0 \\
\delta \Pi_{\text {press }, t}^{\Delta p}+\delta \Pi_{\text {press }, t}^{\Delta \mathbf{n}}=-\delta \Pi_{\text {press }, t}
\end{gathered}
$$

For each enclosed chamber $i$ we get:

$$
\begin{aligned}
& -\frac{\kappa p_{t, i}}{v_{t, i}} \int_{\eta} \int_{\xi} \delta \mathbf{u} \cdot \mathbf{n}_{t}^{*} \mathrm{~d} \xi \mathrm{d} \eta \int_{\eta} \int_{\xi} \mathbf{n}_{t}^{*} \cdot \Delta \mathbf{u} \mathrm{d} \xi \mathrm{d} \eta \quad \text { (volume change) } \\
& +\frac{p_{t, i}}{2} \int_{\eta} \int_{\xi}\left(\begin{array}{c}
\delta \mathbf{u} \\
\delta \mathbf{u}_{, \xi} \\
\delta \mathbf{u}_{, \eta}
\end{array}\right) \cdot\left[\begin{array}{ccc}
0 & \underline{\mathbf{W}^{\xi}} & \underline{\mathbf{W}}^{\eta} \\
\underline{\mathbf{W}}^{\xi \mathrm{T}} & 0 & 0 \\
\underline{\mathbf{W}}^{\eta T} & 0 & 0
\end{array}\right]\left(\begin{array}{c}
\Delta \mathbf{u} \\
\Delta \mathbf{u}_{, \xi} \\
\Delta \mathbf{u}_{, \eta}
\end{array}\right) \mathrm{d} \xi \mathrm{d} \eta \quad \text { (load stiffness matrix) } \\
& =-p_{t, i} \int_{\eta} \int_{\xi} \mathbf{n}_{t}^{*} \cdot \delta \mathbf{u} \mathrm{d} \xi \mathrm{d} \eta \quad(\text { load vector })
\end{aligned}
$$

Thus as expected the conservativeness is confirmed by the symmetric form obtained for each part separately. The first part for the volume change clearly leads to a rank-one update of the stiffness matrix.

As an alternative a mixed formulation is presented in the following with the pressure change $\Delta p$ as a separate unknown, here written for one chamber only. Thus, we have one equation for the gas law, see Equation (35) and the standard set of equations with additional terms for $\Delta p$, see Equation (36).

$$
\begin{aligned}
& \Delta p+\kappa \frac{p_{t}}{v_{t}} \int_{\eta} \int_{\xi} \mathbf{n}_{t}^{*} \cdot \Delta \mathbf{u} \mathrm{d} \xi \mathrm{d} \eta=0 \\
& \Delta p \int_{\eta} \int_{\xi} \mathbf{n}_{t}^{*} \cdot \delta \mathbf{u} \mathrm{d} \xi \mathrm{d} \eta \\
& \quad+\frac{p_{t}}{2} \int_{\eta} \int_{\xi}\left(\begin{array}{c}
\delta \mathbf{u} \\
\delta \mathbf{u}_{, \xi} \\
\delta \mathbf{u}_{, \eta}
\end{array}\right) \cdot\left[\begin{array}{crr}
0 & \underline{\mathbf{W}}^{\xi} & \underline{\mathbf{W}}^{\eta} \\
\underline{\mathbf{W}}^{\xi \mathrm{T}} & 0 & 0 \\
\underline{\mathbf{W}}^{\eta \mathrm{T}} & 0 & 0
\end{array}\right]\left(\begin{array}{c}
\Delta \mathbf{u} \\
\Delta \mathbf{u}_{, \xi} \\
\Delta \mathbf{u}_{, \eta}
\end{array}\right) \mathrm{d} \xi \mathrm{d} \eta
\end{aligned}
$$




$$
=-p_{t} \int_{\eta} \int_{\xi} \mathbf{n}_{t}^{*} \cdot \delta \mathbf{u} \mathrm{d} \xi \mathrm{d} \eta
$$

If this form is taken, it allows a different development concerning the symmetry of the expression, which becomes more visible after the discretization shown in the following.

\section{FE-DISCRETIZATION}

The variation of the potential energy $W$ with $V$ as the elastic potential of the structure and $\delta \Pi$ as the virtual work of the external forces vanishes, see also Section 4.3 , thus

$$
\delta W=\delta V-\delta \Pi=0
$$

Linearizing by means of a Taylor series expansion, we embed the non-linear formulation in a Newton-type scheme as discussed above.

$$
\delta W\left(\mathbf{x}_{t}+\Delta \mathbf{u}\right)=\delta W\left(\mathbf{x}_{t}\right)+D \delta W\left(\mathbf{x}_{t}\right) \Delta \mathbf{u}=0
$$

Equation (38) allows to establish a piecewise linear system of equations:

$$
D\left[\delta V\left(\mathbf{x}_{t}\right)-\delta \Pi\left(\mathbf{x}_{t}\right)\right] \Delta \mathbf{u}=-\left[\delta V\left(\mathbf{x}_{t}\right)-\delta \Pi\left(\mathbf{x}_{t}\right)\right]
$$

If an isoparametric representation is taken for the FE-discretization

$$
\mathbf{x}_{t}=\mathbf{N x} ; \quad \Delta \mathbf{u}=\mathbf{N d} \text { and } \delta \mathbf{u}=\mathbf{N} \delta \mathbf{d}
$$

then the global stiffness matrix and the load vector become

$$
\left[V_{, \mathbf{x x}}-\Pi_{, \mathbf{x x}}\right] \mathbf{d}=-\left(V_{, \mathbf{x}}-\Pi_{, \mathbf{x}}\right)
$$

The coupled problem for the deformation including the volume change is then written in the case of one chamber $i$ as follows, if the alternative formulation is used, see also Equations (35) and (36):

$$
\left[\begin{array}{cc}
\mathbf{K}_{\mathrm{T}}-\mathbf{K}_{\text {press }, i} & -\mathbf{a}_{i} \\
\mathbf{a}_{i}^{\mathrm{T}} & b_{i}^{-1}
\end{array}\right]\left(\begin{array}{c}
\mathbf{d} \\
\Delta p
\end{array}\right)=\left(\begin{array}{c}
\mathbf{f}_{\mathrm{ext}}+\mathbf{f}_{\mathrm{press}, i}-\mathbf{f}_{\mathrm{int}} \\
0
\end{array}\right)
$$

After elimination of $\Delta p$ the symmetric form of the pure displacement formulation becomes again obvious, see also Equation (34).

$$
\begin{aligned}
{\left[\mathbf{K}_{T}-\mathbf{K}_{\text {press }, i}+b_{i} \mathbf{a}_{i} \otimes \mathbf{a}_{i}\right] \mathbf{d} } & =\mathbf{f}_{\text {ext }}+\mathbf{f}_{\text {press }, i}-\mathbf{f}_{\text {int }} \\
\mathbf{K}_{\text {press }, i} & =\frac{p_{t, i}}{2} \sum_{e} \int_{\eta} \int_{\xi}\left(\begin{array}{c}
\mathbf{N} \\
\mathbf{N}_{\xi} \\
\mathbf{N}_{\eta}
\end{array}\right)^{\mathrm{T}}\left[\begin{array}{ccc}
0 & \underline{\mathbf{W}^{\xi}} & \underline{\mathbf{W}}^{\eta} \\
\underline{\mathbf{W}}^{\xi \mathrm{T}} & 0 & 0 \\
\underline{\mathbf{W}}^{\eta \mathrm{T}} & 0 & 0
\end{array}\right]\left(\begin{array}{l}
\mathbf{N} \\
\mathbf{N}_{\xi} \\
\mathbf{N}_{\eta}
\end{array}\right) \mathrm{d} \xi \mathrm{d} \eta \\
\mathbf{a}_{i} & =\sum_{e} \int_{\eta} \int_{\xi} \mathbf{N}^{\mathrm{T}} \mathbf{n}_{t}^{*} \mathrm{~d} \xi \mathrm{d} \eta
\end{aligned}
$$




$$
\begin{aligned}
\mathbf{f}_{\text {press }, i} & =p_{t, i,} \sum_{e} \int_{\eta} \int_{\xi} \mathbf{N}^{\mathrm{T}} \mathbf{n}_{t}^{*} \mathrm{~d} \xi \mathrm{d} \eta \\
b_{i} & =\kappa \frac{p_{t, i}}{v_{t, i}} \\
\mathrm{e} & =\text { number of surface elements describing the chamber } i
\end{aligned}
$$

The global stiffness matrix and the global load vector are obtained by adding the load stiffness matrix $\mathbf{K}_{\text {press }, i}$ and the load vector $\mathbf{f}_{\text {press }, i}$ to the element stiffness matrix $\mathbf{K}_{T}$ containing linear and non-linear terms and to the residuum vector $\mathbf{f}_{\text {int }}$ and the vector of the external forces $\mathbf{f}_{\text {ext }}[9,10]$. The volume dependence leads to a rank-one update of the global stiffness matrix for each closed chamber $i$ thus linking of all variables describing the surface of chamber $i$ as already obtained by Bonet et al. [15]. If a structure consists of $j$ chambers, then we get pressure matrices for each chamber $i$ and due to the volume changes $j$ rank-one updates have to be taken into account.

$$
\left[\mathbf{K}_{T}-\sum_{i=1}^{j}\left(\mathbf{K}_{\text {press }, i}-b_{i} \mathbf{a}_{i} \otimes \mathbf{a}_{i}\right)\right] \mathbf{d}=\mathbf{f}_{\mathrm{ext}}+\sum_{i=1}^{j} \mathbf{f}_{\text {press }, i}-\mathbf{f}_{\text {int }}
$$

\section{SOLUTION ALGORITHM}

As already shown by Bonet et al. [15] without restriction of any kind Equation (43) can be rewritten for one chamber as follows:

$$
\left[\mathbf{K}^{*}+b \mathbf{a} \otimes \mathbf{a}\right] \mathbf{d}=\mathbf{F}
$$

with $\mathbf{K}^{*}=\mathbf{K}_{T}-\mathbf{K}_{\text {press }}$ and $\mathbf{F}=\mathbf{f}_{\text {ext }}+\mathbf{f}_{\text {press }}-\mathbf{f}_{\text {int }}$.

According to Sherman-Morrison [19] the solution of such a problem can be achieved by:

$$
\begin{aligned}
\mathbf{d} & =\mathbf{K}^{*-1} \mathbf{F}-\beta \mathbf{r} \otimes \mathbf{r F} \\
\text { with } \Delta \mathbf{u}_{\mathrm{I}} & =\mathbf{K}^{*-1} \mathbf{F} \\
\mathbf{r} & =\mathbf{K}^{*-1} \mathbf{a} \equiv \Delta \mathbf{u}_{\mathrm{II}} \\
\text { and } \quad \beta & =\frac{b}{1+b \mathbf{a} \cdot \mathbf{r}}
\end{aligned}
$$

Introducing Equations (52)-(54) into Equation (51) the solution vector $\mathbf{d}$ can be obtained by one additional forward-backward substitution for each chamber plus two scalar products within the solution process using a direct solver.

$$
\mathbf{d}=\Delta \mathbf{u}_{\mathrm{I}}-\frac{b \Delta \mathbf{u}_{\mathrm{II}} \cdot \mathbf{F}}{1+b \mathbf{a} \cdot \Delta \mathbf{u}_{\mathrm{II}}} \Delta \mathbf{u}_{\mathrm{II}}
$$

It must be noted that in the input phase a specification of all loaded surface segments, their relation to closed volumes and their normal direction is necessary. Within the algorithm the 


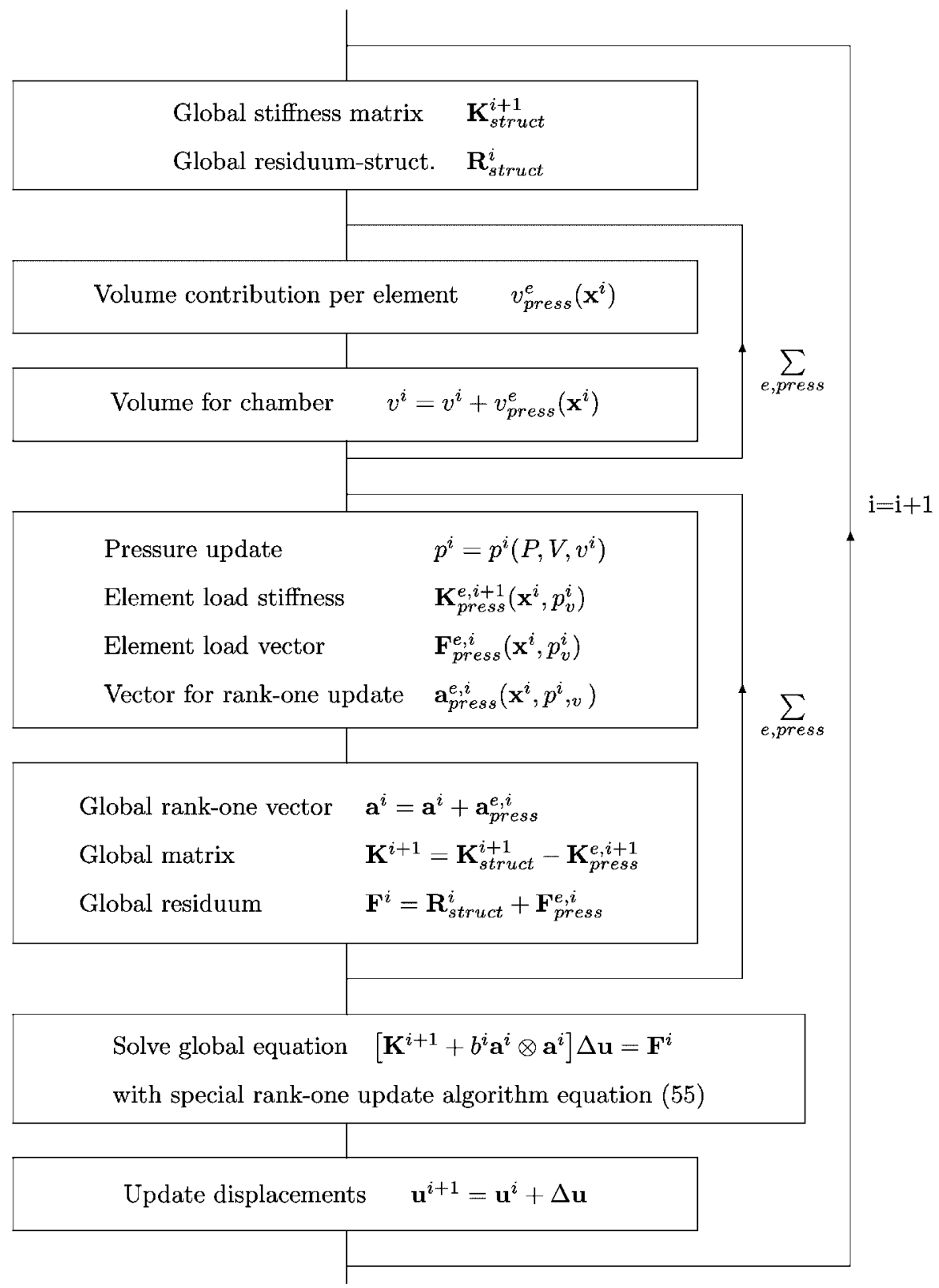

Figure 4. Set-up of stiffness matrices and residuum vectors for one chamber filled with gas within a Newton-type scheme

actual volume of each chamber has to be computed in order to get the corresponding pressure in the chamber which is a constant in the terms for the load stiffness matrix and the load vector, see Figure 4. 


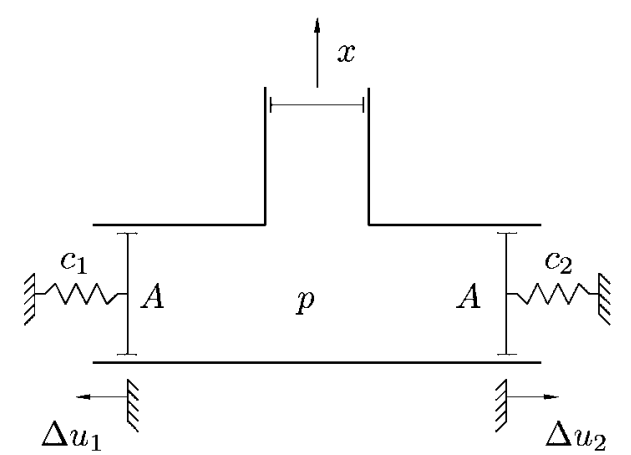

Figure 5. Piston controlled chamber with two springs supporting the walls.

\section{VOLUME EFFECTS ON THE STABILITY OF STRUCTURES}

It is physically obvious, that the linkage of all variables by the thermodynamic gas equation leads to a stabilization of the system, indicated by a higher value of the determinant and a higher first eigenvalue $\lambda$ of the stiffness matrix, illustrated by a simple example, see Figure 5 . A rigid chamber with two springs supporting the walls (spring stiffnesses $c_{1}, c_{2}$; surface of the walls $A$ ) is pressurized by a piston controlled motion $x$, thus a controlled volume. For the global stiffness matrix, the determinant and the eigenvalues become:

$$
\begin{aligned}
\mathbf{K}^{*} & =\left[\begin{array}{ll}
c_{1} & 0 \\
0 & c_{2}
\end{array}\right]+\kappa \frac{p_{t}}{v_{t}}\left(\begin{array}{l}
A \\
A
\end{array}\right)(A, A) \\
\operatorname{det} \mathbf{K}^{*} & =c_{1} c_{2}+\kappa \frac{p_{t}}{v_{t}} A^{2}\left(c_{1}+c_{2}\right) \\
\lambda_{1,2} & =\frac{1}{2}\left[c_{1}+c_{2}+2 \kappa \frac{p_{t}}{v_{t}} A^{2} \pm \sqrt{\left(c_{1}-c_{2}\right)^{2}+\left(2 \kappa \frac{p_{t}}{v_{t}} A^{2}\right)^{2}}\right]
\end{aligned}
$$

In the case $c_{1}=c_{2}=c$ this simplifies to

$$
\begin{aligned}
\operatorname{det} \mathbf{K}^{*} & =c^{2}+2 \kappa \frac{p_{t}}{v_{t}} A^{2} c \\
\lambda_{1} & =c ; \quad \lambda_{2}=c+2 \kappa \frac{p_{t}}{v_{t}} A^{2}
\end{aligned}
$$

\section{NON-LINEAR ANALYSIS USING ARC-LENGTH PROCEDURES}

Two special cases are investigated following the methods developed in References [2, 20-23] in order to distinguish between two major technically important cases, which have some implications on the solution process. In the first case the focus is on geometrically 
non-linear structures with closed chambers loaded by an external loading, controlled by arclength procedures. In addition for each chamber Poisson's law is satisfied.

In the second case an arc-length controlled external displacement e.g. a piston motion modifies an initial volume resulting in a pressure change of a closed structure. For both cases the necessary equations for an implementation of arc-length methods in connection with volume-dependent pressure loadings are prepared and illustrated by simple examples.

\subsection{Arc-length controlled loading on pressurized closed structures}

The expression for the external virtual work of an external force $\mathbf{f}_{\text {ext }}$ with the control quantity $\lambda$ and a pressure load can be written as

$$
\delta \Pi_{\mathrm{ext}}=\lambda \mathbf{f}_{\mathrm{ext}} \cdot \delta \mathbf{u}+\int_{\eta} \int_{\xi} p \mathbf{n} \cdot \delta \mathbf{u} \mathrm{d} \xi \mathrm{d} \eta
$$

The physical behaviour of the enclosed gas is specified by Poisson's law

$$
p v^{\kappa}=P V^{\kappa}
$$

Introducing a path following algorithm an arbitrary function of displacement and load level is needed as control law

$$
g(\mathbf{x}, \lambda)=0
$$

After the discretization of the potential energy $\delta W=\delta V-\delta \Pi$ with the virtual work expression $\delta \Pi$ of Equation (61) and the additional Equations (62), (63) the linearization at state $t$ for an incremental iterative procedure with the load level $\lambda$ as an additional unknown yields:

$$
\left[\begin{array}{ccc}
\mathbf{K}_{T}-\mathbf{K}_{\text {press }} & -\mathbf{a} & -\mathbf{f}_{\mathrm{ext}} \\
\mathbf{a}^{\mathrm{T}} & b^{-1} & 0 \\
\mathbf{z}^{\mathrm{T}} & 0 & \alpha
\end{array}\right]\left(\begin{array}{c}
\mathbf{d} \\
\Delta p \\
\Delta \lambda
\end{array}\right)=\left(\begin{array}{c}
\lambda \mathbf{f}_{\mathrm{ext}}+\mathbf{f}_{\text {press }}-\mathbf{f}_{\mathrm{int}} \\
0 \\
-g
\end{array}\right)
$$

With the abbreviations for the directional derivatives:

$$
\begin{aligned}
& \mathbf{z}=\left.\frac{\mathrm{d}}{\mathrm{d} \varepsilon} g\left(\mathbf{x}_{t}+\varepsilon \Delta \mathbf{u}, \lambda_{t}\right)\right|_{\varepsilon=0} \\
& \alpha=\left.\frac{\mathrm{d}}{\mathrm{d} \varepsilon} g\left(\mathbf{x}_{t}, \lambda_{t}+\varepsilon \Delta \lambda\right)\right|_{\varepsilon=0}
\end{aligned}
$$

Via the elimination of $\Delta p$ the system of equations can be reduced

$$
\left[\begin{array}{cc}
\mathbf{K}^{*}+b \mathbf{a} \otimes \mathbf{a}-\mathbf{P} \\
\mathbf{z}^{\mathrm{T}} & \alpha
\end{array}\right]\left(\begin{array}{c}
\mathbf{d} \\
\Delta \lambda
\end{array}\right)=\left(\begin{array}{c}
\mathbf{R}+\lambda \mathbf{P} \\
-g
\end{array}\right)
$$

For simplicity $\mathbf{R}=\mathbf{f}_{\text {press }}-\mathbf{f}_{\text {int }}$ as residuum of the internal forces and $\mathbf{P}=\mathbf{f}_{\text {ext }}$ as external force is introduced. After a further elimination of $\Delta \lambda$ the problem is once again reduced into a 
pure displacement formulation

$$
\left[\mathbf{K}^{*}+b \mathbf{a} \otimes \mathbf{a}+\frac{1}{\alpha} \mathbf{P} \otimes \mathbf{z}\right] \mathrm{d}=\mathbf{F}-\frac{g}{\alpha} \mathbf{P}
$$

It is obvious that the global stiffness matrix $\mathbf{K}^{*}$ is finally updated by two rank-one matrices. The right-hand side $\mathbf{F}=\mathbf{R}+\lambda \mathbf{P}$ shows a modification due to the residual of the constraint equation. Applying the Sherman-Morrison formula four times the solution of the modified system of equations can be performed in the following fashion. First $\mathbf{d}$ is splitted into two parts via:

$$
\mathbf{d}=\Delta \lambda \mathbf{d}_{\mathrm{I}}+\mathbf{d}_{\mathrm{II}}
$$

with

$$
\begin{aligned}
\mathbf{d}_{\mathrm{I}} & =\left[\mathbf{K}^{*}+b \mathbf{a} \otimes \mathbf{a}\right]^{-1} \mathbf{P} \\
\mathbf{d}_{\mathrm{II}} & =\left[\mathbf{K}^{*}+b \mathbf{a} \otimes \mathbf{a}\right]^{-1} \mathbf{F} \\
\Delta \lambda & =-\frac{g+\mathbf{z} \cdot \mathbf{d}_{\mathrm{II}}}{\alpha+\mathbf{z} \cdot \mathbf{d}_{\mathrm{I}}}
\end{aligned}
$$

The interim solution vectors $\mathbf{d}_{\mathrm{I}}$ and $\mathbf{d}_{\mathrm{II}}$ are computed via:

$$
\begin{aligned}
\mathbf{d}_{\mathrm{I}} & =\mathbf{u}_{\mathrm{I}}-\frac{b \mathbf{u}_{\mathrm{II}} \cdot \mathbf{P}}{1+b \mathbf{a} \cdot \mathbf{u}_{\mathrm{II}}} \mathbf{u}_{\mathrm{II}} \\
\mathbf{d}_{\mathrm{II}} & =\mathbf{u}_{\mathrm{III}}-\frac{b \mathbf{u}_{\mathrm{II}} \cdot \mathbf{F}}{1+b \mathbf{a} \cdot \mathbf{u}_{\mathrm{II}}} \mathbf{u}_{\mathrm{II}}
\end{aligned}
$$

whereby

$$
\begin{aligned}
\mathbf{u}_{\mathrm{I}} & =\mathbf{K}^{*-1} \mathbf{P} \\
\mathbf{u}_{\mathrm{II}} & =\mathbf{K}^{*-1} \mathbf{a} \\
\mathbf{u}_{\mathrm{III}} & =\mathbf{K}^{*-1} \mathbf{F}
\end{aligned}
$$

As it can be seen, the solution vector $\mathbf{d}$ requires three forward-backward substitutions within one step of the iterative solution process.

8.1.1. Non-linear mechanism with gas chamber and arc-length controlled external loading. For illustration purposes a simple example is chosen. An external force $\lambda f$ is applied via a slider plunger mechanism on a gas chamber, see Figure 6 . The virtual work and the gas law of the system are formulated in the independent variables, angle $\varphi$ and load level $\lambda$ :

$$
\begin{aligned}
\delta W=-\delta \Pi=[(P-p) s l \sin \varphi+\lambda f l \cos \varphi] \delta \varphi & =0 \\
p\left[V-s l\left(\cos \varphi-\cos \varphi_{0}\right)\right]^{\kappa} & =P V^{\kappa}
\end{aligned}
$$




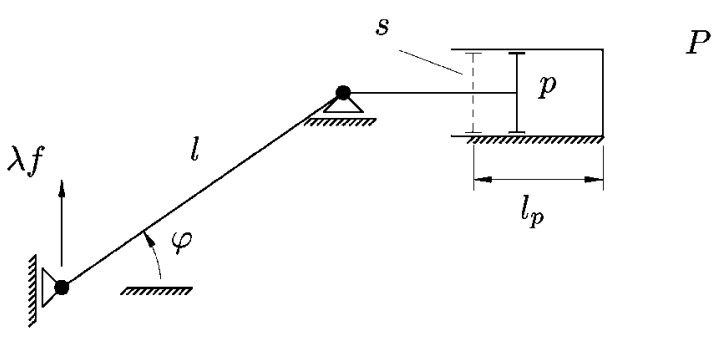

Figure 6. Non-linear slider-plunger mechanism with gas chamber and arc-length controlled loading.

Introducing a non-dimensional formulation with

$$
\psi=\frac{p}{P}, \quad \Phi=\frac{f}{P s}, \quad \Lambda=\frac{s l}{V}=\frac{l}{l_{p}}
$$

leads to:

$$
\begin{array}{r}
(1-\psi) \sin \varphi+\lambda \Phi \cos \varphi=0 \\
\psi\left[1-\Lambda\left(\cos \varphi-\cos \varphi_{0}\right)\right]^{\kappa}=1
\end{array}
$$

As control law an iteration on normal surfaces [23] with $\mathbf{t}$ as tangent vector on the load deflection curve is chosen

$$
\begin{aligned}
g & =\mathbf{t}_{t} \cdot\left(\mathbf{t}-\mathbf{t}_{t}\right) \\
& =\varphi_{t}\left(\varphi-\varphi_{t}\right)+\lambda_{t} \Phi^{2}\left(\lambda-\lambda_{t}\right)
\end{aligned}
$$

After linearizing the equations for an iterative procedure the necessary coefficients according to Section 8.1 are:

$$
\begin{aligned}
K^{*} & =\left(1-\psi_{t}\right) \cos \varphi_{t}-\lambda_{t} \Phi \sin \varphi_{t}, & a=\sin \varphi_{t}, & z=\varphi_{t} \\
P & =-\Phi \cos \varphi_{t}, & b=\frac{\kappa \psi_{t} \Lambda}{1-\Lambda\left(\cos \varphi_{t}-\cos \varphi_{0}\right)} & \alpha=\Phi^{2} \lambda_{t} \\
R & =\left(1-\psi_{t}\right) \sin \varphi_{t} & &
\end{aligned}
$$

Following the known algorithm with predictor and corrector steps the load- $\varphi$ curve in Figure 7 can be computed.

\subsection{Arc-length controlled displacement loading on pressurized closed structures}

Several technical applications e.g. in pneumatic systems demand a finite volume change. A genuine rendition requires an arc-length controlled displacement. Then the expression for the virtual work expression remains unaltered

$$
\delta \Pi_{\mathrm{ext}}=\int_{\eta} \int_{\xi} p \mathbf{n} \cdot \delta \mathbf{u} \mathrm{d} \xi \mathrm{d} \eta
$$




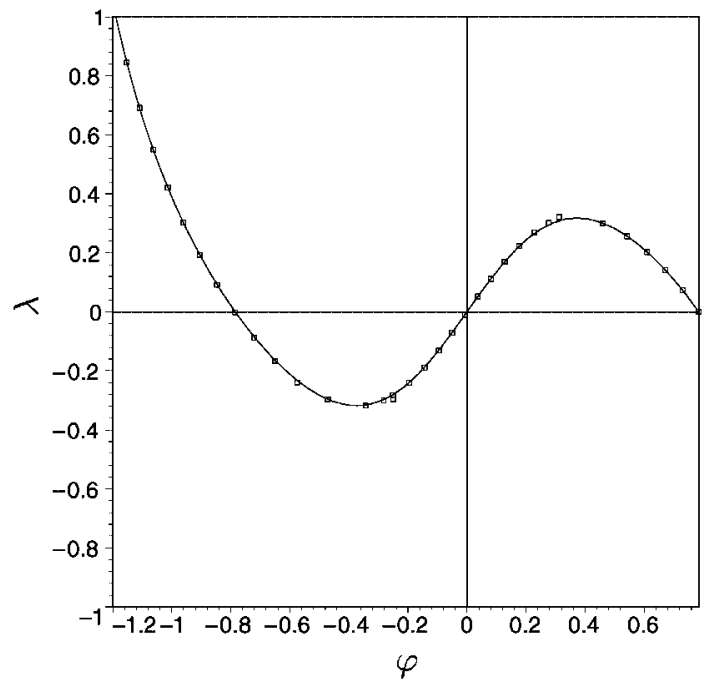

Figure 7. Non-linear slider-plunger mechanism with gas chamber and arc-length controlled loading; load- $\varphi$ curve; - : exact solution, $\square$ : iterative solution; $\Lambda=2, \varphi_{0}=\pi / 4, \kappa=1, \Phi=1$.

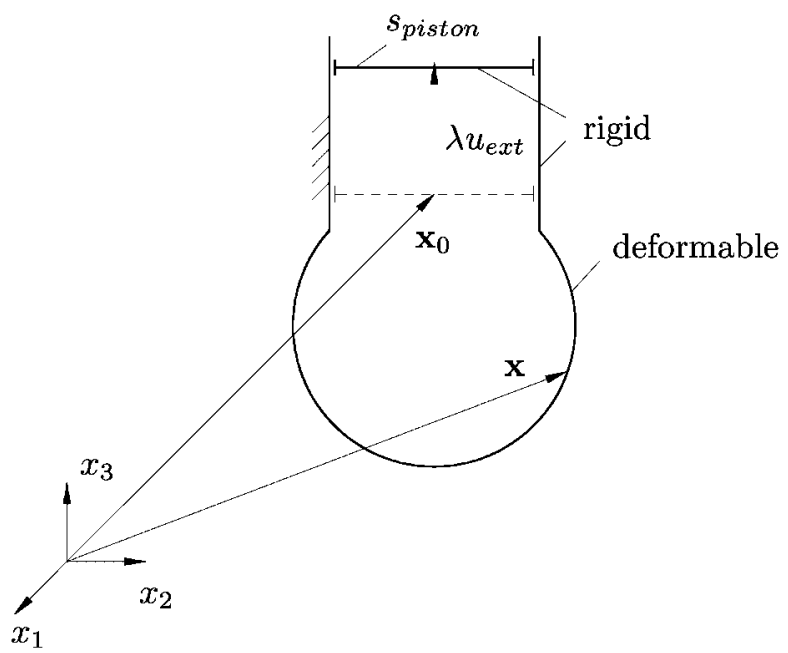

Figure 8. Volume computation; displacement controlled loading.

However, Poisson's law especially the volume computation is modified by the controlled displacement

$$
p \underline{v}(\lambda)^{\kappa}=P V^{\kappa}
$$

whereby the volume $\underline{v}(\lambda)$ of the whole structure is decomposed into a deformable part and into a rigid part, see Figure 8 . As a consequence the volume computation is separated into 
two terms, the deformable structure $v_{\text {struct }}$ and the rigid structure, the piston volume $v_{\text {piston }}$.

$$
\begin{aligned}
& \underline{v}(\lambda)=v_{\text {struct }}+\lambda v_{\text {piston }} \\
& \underline{v}(\lambda)=\frac{1}{3} \int_{\eta} \int_{\xi}\left(\mathbf{x}-\mathbf{x}_{0}\right) \cdot \mathbf{n}^{*} \mathrm{~d} \xi \mathrm{d} \eta+s_{\text {piston }} \lambda u_{\text {ext }}
\end{aligned}
$$

As in the preceeding sections the additional constraint for the control of the load level $\lambda$ and the displacement $u_{\text {ext }}$ is

$$
g(\mathbf{x}, \lambda)=0
$$

After the linearization of the potential energy $\delta W=\delta V-\delta \Pi$, and of Poisson's law in Equation (86) and of the constraint Equation (89) a discretization leads to the following set of equations, following Equation (64):

$$
\left[\begin{array}{ccc}
\mathbf{K}_{T}-\mathbf{K}_{\text {press }} & -\mathbf{a} & 0 \\
\mathbf{a}^{\mathrm{T}} & \underline{b}^{-1}(\lambda) & c \\
\mathbf{z}^{\mathrm{T}} & 0 & \alpha
\end{array}\right]\left(\begin{array}{c}
\mathbf{d} \\
\Delta p \\
\Delta \lambda
\end{array}\right)=\left(\begin{array}{c}
\mathbf{f}_{\text {press }}-\mathbf{f}_{\mathrm{int}} \\
0 \\
-g
\end{array}\right)
$$

whereby

$$
\begin{aligned}
\underline{b}(\lambda) & =\frac{\kappa p_{t}}{\underline{v}_{t}(\lambda)} \\
c & =v_{\text {piston }}=S_{\text {piston }} u_{\mathrm{ext}} \\
\text { with } \quad \underline{v}_{t}(\lambda) & =\frac{1}{3} \sum_{e} \int_{\eta} \int_{\xi}\left(\mathbf{x}_{t}-\mathbf{x}_{0}\right) \cdot \mathbf{n}_{t}^{*} \mathrm{~d} \xi \mathrm{d} \eta+\lambda_{t} v_{\text {piston }}
\end{aligned}
$$

Reducing the system of equations by eliminating $\Delta p$, the standard set of equations for an arc-length method is once again obtained, with $\mathbf{R}=\mathbf{f}_{\text {press }}-\mathbf{f}_{\text {int }}$ as residuum of the internal forces and $\mathbf{P}(\lambda)=-\underline{b}(\lambda) c \mathbf{a}$ as vector of the external forces.

$$
\left[\begin{array}{cc}
\mathbf{K}^{*}+\underline{b}(\lambda) \mathbf{a} \otimes \mathbf{a} & -\mathbf{P}(\lambda) \\
\mathbf{z}^{\mathrm{T}} & \alpha
\end{array}\right]\left(\begin{array}{c}
\mathbf{d} \\
\Delta \lambda
\end{array}\right)=\left(\begin{array}{c}
\mathbf{R} \\
-g
\end{array}\right)
$$

It should be noted that the vector of the external forces $\mathbf{P}(\lambda)$ is not linear concerning the control quantity $\lambda$. Finally, a further reduction of the system as shown in Equation (68) leads to the pure displacement formulation

$$
\left[\mathbf{K}^{*}+\underline{b}(\lambda) \mathbf{a} \otimes \mathbf{a}+\frac{1}{\alpha} \mathbf{P}(\lambda) \otimes \mathbf{z}\right] \mathbf{d}=\mathbf{R}-\frac{g}{\alpha} \mathbf{P}(\lambda)
$$

Computing the solution vector $\mathbf{d}$ via

$$
\mathbf{d}=\Delta \lambda \mathbf{d}_{\mathrm{I}}+\mathbf{d}_{\mathrm{II}}
$$




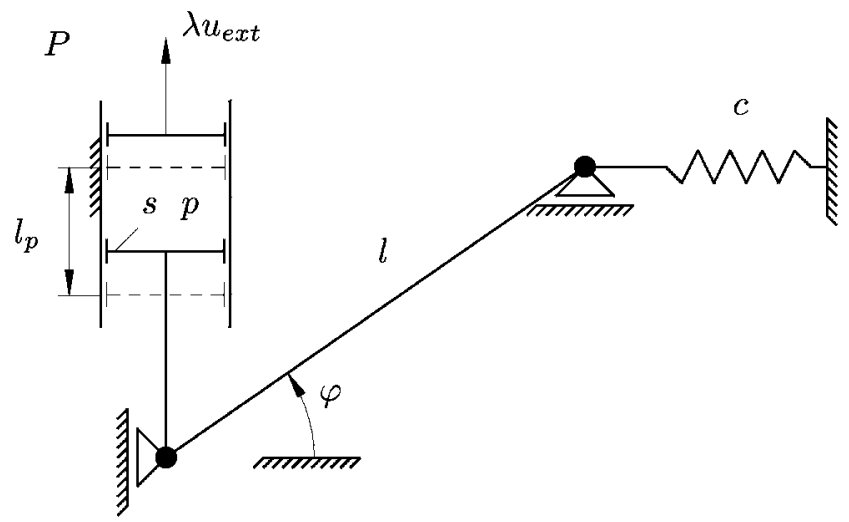

Figure 9. Non-linear slider-plunger mechanism with gas chamber and arc-length controlled displacement.

with

$$
\begin{aligned}
\mathbf{d}_{\mathrm{I}} & =\left[\mathbf{K}^{*}+\underline{b} \mathbf{a} \otimes \mathbf{a}\right]^{-1} \mathbf{P} \stackrel{!}{=}-\underline{b} c\left[\mathbf{K}^{*}+\underline{b} \mathbf{a} \otimes \mathbf{a}\right]^{-1} \mathbf{a} \\
\mathbf{d}_{\mathrm{II}} & =\left[\mathbf{K}^{*}+\underline{b} \mathbf{a} \otimes \mathbf{a}\right]^{-1} \mathbf{R} \\
\Delta \lambda & =-\frac{g+\mathbf{z} \cdot \mathbf{d}_{\mathrm{II}}}{\alpha+\mathbf{z} \cdot \mathbf{d}_{\mathrm{I}}}
\end{aligned}
$$

it can be seen that only two forward-backward substitutions within the iterative solution process are necessary. Then the interim solution vectors are

$$
\begin{aligned}
\mathbf{d}_{\mathrm{I}} & =-\frac{\underline{b} c+2 \underline{b}^{2} c \mathbf{a} \cdot \mathbf{u}_{\mathrm{II}}}{1+\underline{b} \mathbf{a} \cdot \mathbf{u}_{\mathrm{II}}} \mathbf{u}_{\mathrm{II}} \\
\mathbf{d}_{\mathrm{II}} & =\mathbf{u}_{\mathrm{III}}-\frac{\underline{b} \mathbf{u}_{\mathrm{II}} \cdot \mathbf{R}}{1+\underline{b} \mathbf{a} \cdot \mathbf{u}_{\mathrm{II}}} \mathbf{u}_{\mathrm{II}}
\end{aligned}
$$

with

$$
\begin{aligned}
\mathbf{u}_{\mathrm{II}} & =\mathbf{K}^{*-1} \mathbf{a} \\
\mathbf{u}_{\mathrm{III}} & =\mathbf{K}^{*-1} \mathbf{R}
\end{aligned}
$$

8.2.1. Nonlinear slider-plunger mechanism with gas chamber and arc-length controlled displacement. For the illustration a very simple example is chosen. An arc-length controlled piston motion acts on a spring supported (spring stiffness $c$ ) slider-plunger mechanism via a gas chamber, see Figure 9. The virtual work of the system and the gas law are formulated in the independent variables, angle $\varphi$ and load level $\lambda$ :

$$
\begin{aligned}
\delta W=\delta V-\delta \Pi=\left[-c l^{2}\left(\cos \varphi-\cos \varphi_{0}\right) \sin \varphi+(P-p) s l \cos \varphi\right] \delta \varphi & =0 \\
p\left[V-\operatorname{sl}\left(\sin \varphi_{0}-\sin \varphi\right)+\lambda s u_{\mathrm{ext}}\right]^{\kappa} & =P V^{\kappa}
\end{aligned}
$$


Introducing a non-dimensional formulation with

$$
\psi=\frac{p}{P}, \quad \eta=\frac{u_{\mathrm{ext}}}{l}, \quad \Lambda=\frac{s l}{V}=\frac{l}{l_{p}}, \quad v=\frac{c l}{P S}
$$

leads to:

$$
\begin{array}{r}
v\left(\cos \varphi-\cos \varphi_{0}\right) \sin \varphi+(1-\psi) \cos \varphi=0 \\
\psi\left[1-\Lambda\left(\sin \varphi_{0}-\sin \varphi\right)+\lambda \Lambda \eta\right]^{\kappa}=1
\end{array}
$$

Like in the preceeding example in Section 8.1.1 an iteration on normal surfaces with $\mathbf{t}$ as tangent vector on the load deflection curve is chosen as constraint equation.

$$
\begin{aligned}
g & =\mathbf{t}_{t} \cdot\left(\mathbf{t}-\mathbf{t}_{t}\right) \\
& =\varphi_{t}\left(\varphi-\varphi_{t}\right)+\lambda_{t} \eta^{2}\left(\lambda-\lambda_{t}\right)
\end{aligned}
$$

After linearizing the equations for an iterative procedure the necessary coefficients according to Section 8.2 become:

$$
\begin{array}{rlrl}
K^{*} & =v \sin \varphi_{t}^{2}-v\left(\cos \varphi_{t}-\cos \varphi_{0}\right) \cos \varphi_{t}-\left(1-\psi_{t}\right) \sin \varphi_{t}, & P=-\underline{b} c a \\
R & =\left(1-\psi_{t}\right) \cos \varphi_{t}-v\left(\cos \varphi_{t}-\cos \varphi_{0}\right) \sin \varphi_{t}, & & a=\cos \varphi_{t} \\
\underline{b} & =\frac{\kappa \psi_{t} \Lambda}{1-\Lambda\left(\sin \varphi_{0}-\cos \varphi_{t}\right)+\lambda \Lambda \eta}, & c & =\eta \\
z & =\varphi_{t}, & \alpha & =\eta^{2} \lambda_{t}
\end{array}
$$

Following the known algorithm with predictor and corrector steps the load- $\varphi$ curve shown in Figure 10 can be computed. The snap-through type of behaviour is clearly visible.

\section{NUMERICAL EXAMPLES}

\subsection{Inflation of a tube with partially folded walls}

The example is chosen to show the effect of the volume change on the pressure-deformation behaviour. An elastic tube with partially folded walls (elastic modulus $E=2.5 \times 10^{5} \mathrm{~N} / \mathrm{cm}^{2}$, Poisson's ratio $v=0.3$, wall thickness $t=0.05 \mathrm{~cm}$ ), connected to a rigid cylinder, is loaded by a displacement controlled piston, see Figure 11 (volume controlled problem). By a downward motion of the piston in the rigid part thus reducing the volume, the pressure increases from 1 to 11 bar, the temperature computed by Poisson's law from 10 to $290^{\circ} \mathrm{C}$ and the complete volume decreases from $6.5 \times 10^{3}$ to $1.1 \times 10^{3} \mathrm{~cm}^{3}$, see Figure 12 . The volume contained by the elastic part of the system is deformed and increased by means of the increased pressure, see Figure 11. The structure undergoes large deformations, which entail a geometrically non-linear approach. Poisson's law used for the temperature-volume computation is

$$
T v^{\kappa-1}=\mathrm{const}
$$




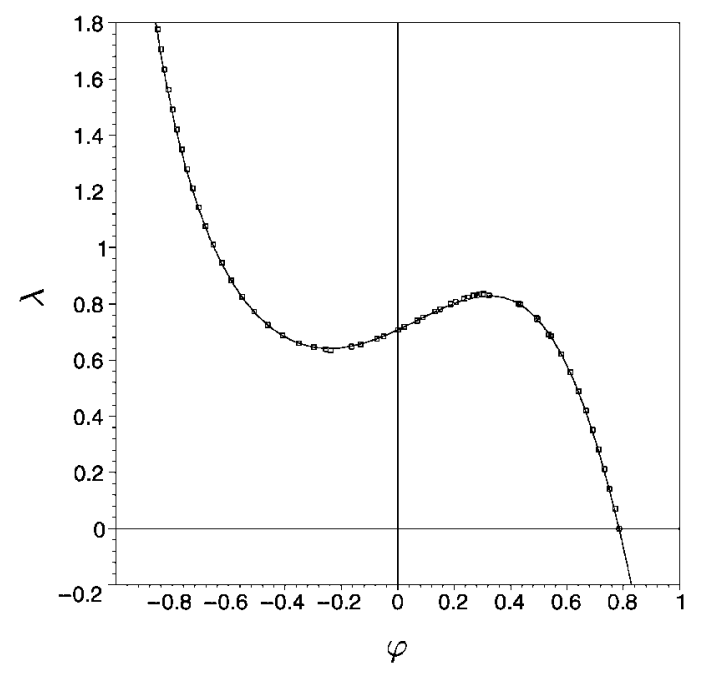

Figure 10. Non-linear slider-plunger mechanism with gas chamber and arc-length controlled displacement; load- $\varphi$ curve; -: exact solution, $\square$ : iterative solution; $v=1, \kappa=1, \Lambda=0.2, \varphi_{0}=\pi / 4$.

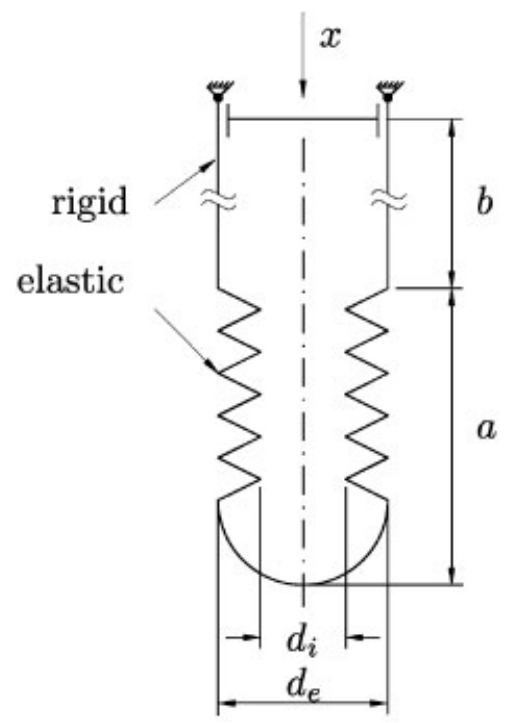

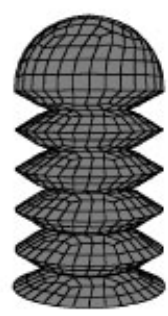

undeformed

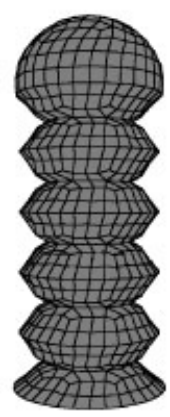

deformed

Figure 11. Inflation of a tube with partially folded walls; loading process (left); undeformed and deformed states (right); $a=15 \mathrm{~cm}, b=75 \mathrm{~cm}, d_{i}=5 \mathrm{~cm}, d_{e}=10 \mathrm{~cm}$.

\subsection{Double chamber structure with different internal pressure loaded by torsion}

This example is chosen to show the effect of the volume consideration first on the interaction between two pressurized chambers and second the stiffening effect of a pressurized 

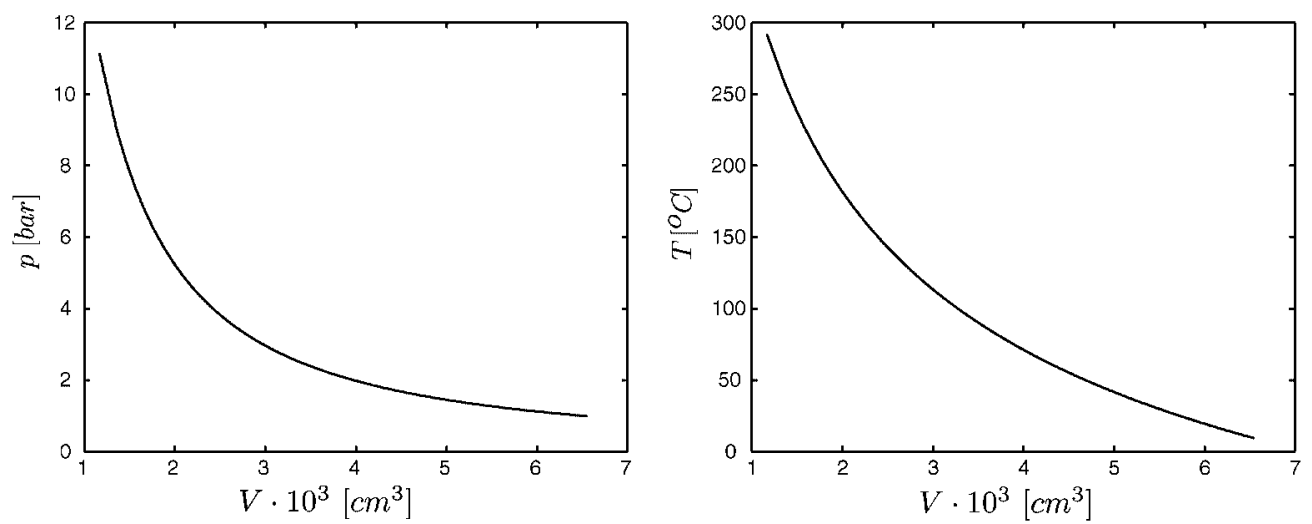

Figure 12. Inflation of a tube with partially folded walls; pressure-volume deformation curve; temperature-volume deformation curve.

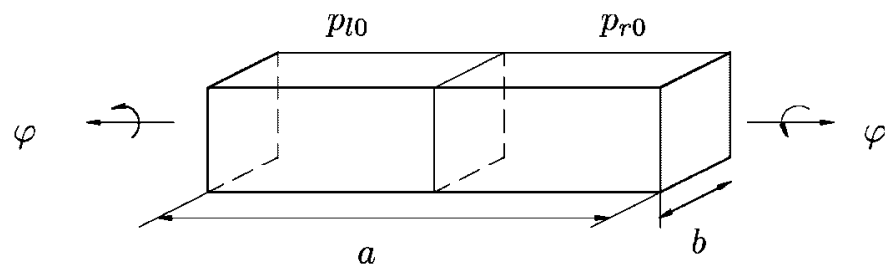

Figure 13. Two chamber system with elastic walls and different internal pressure; loaded by torsion; $a=10 \mathrm{~cm}, b=2 \mathrm{~cm}, \varphi=45^{\circ}, p_{l 0}=0.01$ bar, $p_{r 0}=1$ bar.

chamber. The structure consisting of two chambers which are first loaded with different internal pressure-left with $p_{l 0}=0.01$ bar and right with $p_{r 0}=1$ bar, (elastic modulus $E=$ $2.4 \times 10^{4} \mathrm{~N} / \mathrm{cm}^{2}$, Possion's ratio $v=0.3$, wall thickness $t=0.1 \mathrm{~cm}$ ) see Figures $13-15$-is finally externally loaded by torsion, with a prescribed rotation of the ends. The uniform FE mesh consists of 2300 solid shell elements [24]. The left chamber with the low pressure is deforming heavily, whereas the right chamber with the high pressure shows almost a rigid-body rotation only. The stiffening due to the higher pressure is clearly visible. The pressure deformation curves for both chambers under torsional loading show the influence of the volume consideration directly. The left chamber pressure is continuously increasing with the deformations reducing the volume, whereas the curve for the right chamber shows-indicated by arrows - first some unloading thus decreasing pressure and with increasing torsion and as a consequence a slightly decreasing volume an increasing pressure.

\subsection{Clamped shell under arc-length controlled loading}

The upper shell of a closed container is loaded by a non-concentric single load (elastic modulus $E=1.0 \times 10^{5} \mathrm{~N} / \mathrm{cm}^{2}$, Possion's ratio $v=0.3$, shell thickness $t=0.1 \mathrm{~cm}$ ), see Figure 16 . The loaded shell is clamped on the two straight sides whereas the other sides are allowed to slide in vertical direction. The other walls of the container are assumed as rigid. The 

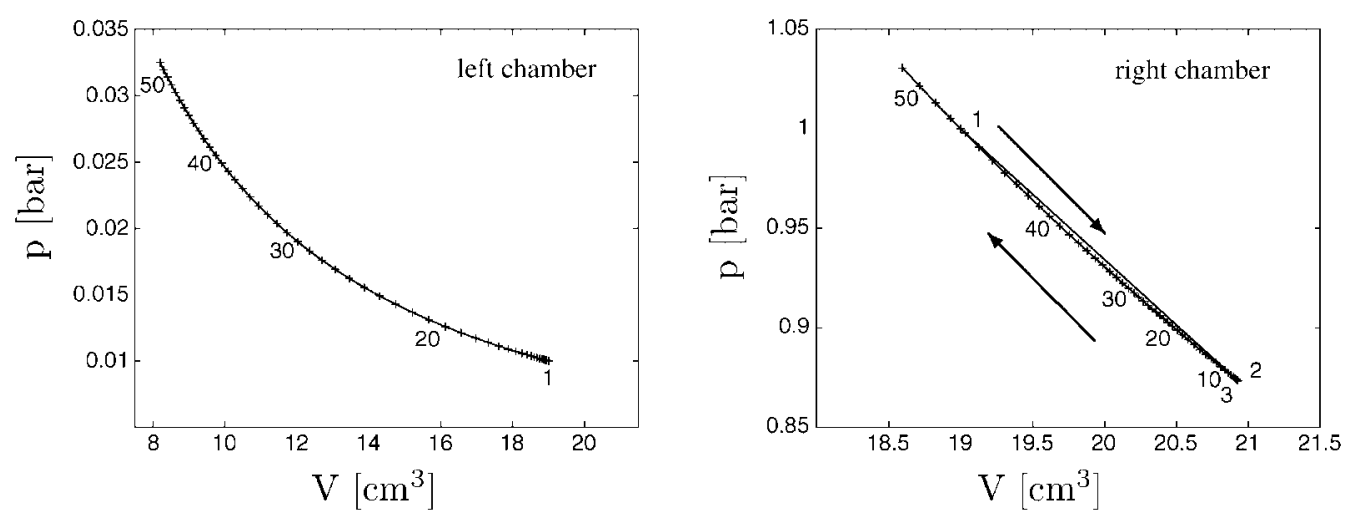

Figure 14. Two chamber system with elastic walls and different internal pressure; external loading by torsion; pressure-volume deformation curves, left chamber (left), right chamber (right).
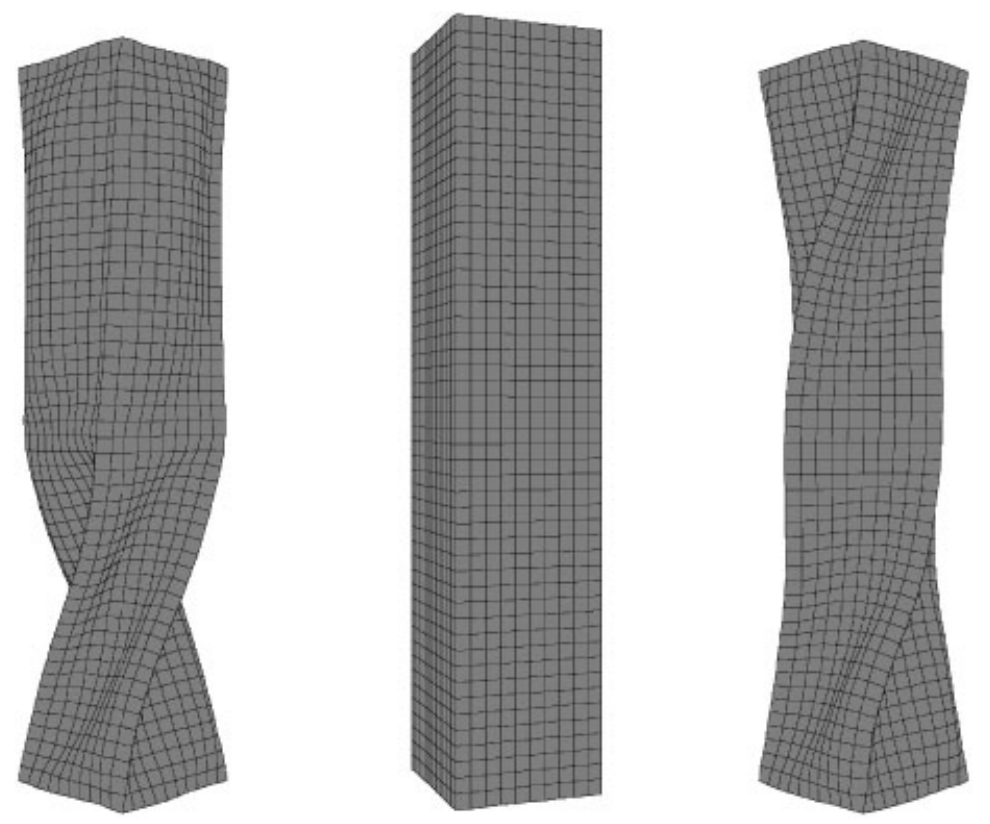

Figure 15. Two chamber system with elastic walls and different internal pressure; external loading by torsion; deformed system with internal pressure (left), undeformed system (centre), deformed system with no internal pressure (right).

external load is algorithmically controlled via the arc-length procedure described in Section 8.1. Owing to the deformation of the upper shell the internal pressure of the container increases, leading to an increase of the critical load of the structure, see Figure 17. A typical snapthrough behaviour is obtained. For comparison only a shell without internal pressure was also computed. Figure 17 shows that the critical loads of the shell with internal pressure 

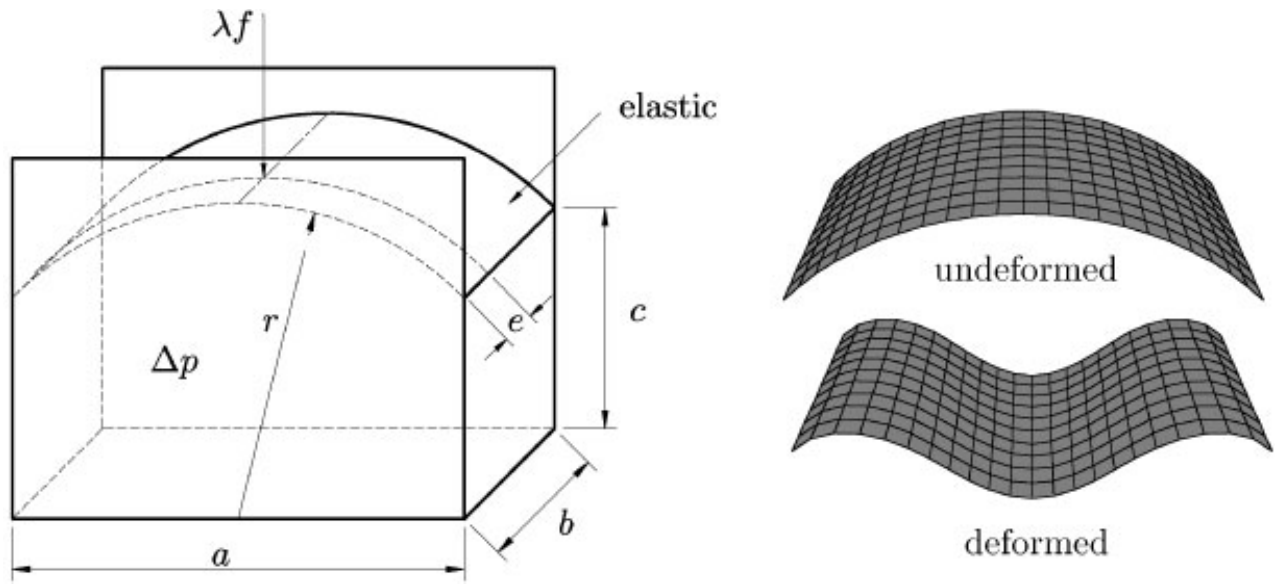

deformed

Figure 16. Closed container with clamped shell under single load; $a=10 \mathrm{~cm}, b=5 \mathrm{~cm}, c=5 \mathrm{~cm}, r=7 \mathrm{~cm}$, $e=2.5 \mathrm{~cm}, f=600 \mathrm{~N}, \lambda$ load factor, $\Delta p$ overpressure; deformed and undeformed shell (right).

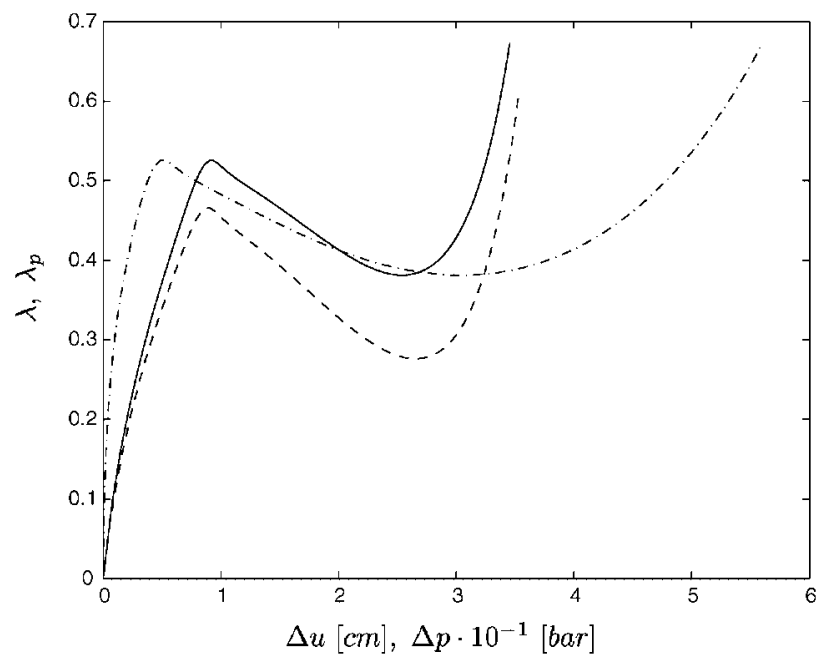

Figure 17. Closed container with clamped shell under controlled loading; $-: \lambda_{p}(\Delta u)$ load-deflection curve with internal pressure, $--: \lambda(\Delta u)$ load-deflection curve without internal pressure, $-\cdot-\lambda_{p}(\Delta p)$ load-overpressure curve, $\Delta u$ deflection of point of application.

(stabilized) and the shell without internal pressure are almost at the same critical deflection, however the internal pressure provides some further resistance.

\subsection{Clamped shell with imperfect geometry under arc-length controlled loading via the motion of a piston}

The bottom shell (elastic modulus $E=1.0 \times 10^{4} \mathrm{~N} / \mathrm{cm}^{2}$, Possion's ratio $v=0.3$, shell thickness $t=0.1 \mathrm{~cm})$ of a closed container is loaded by pressure, created by a downward piston motion of 

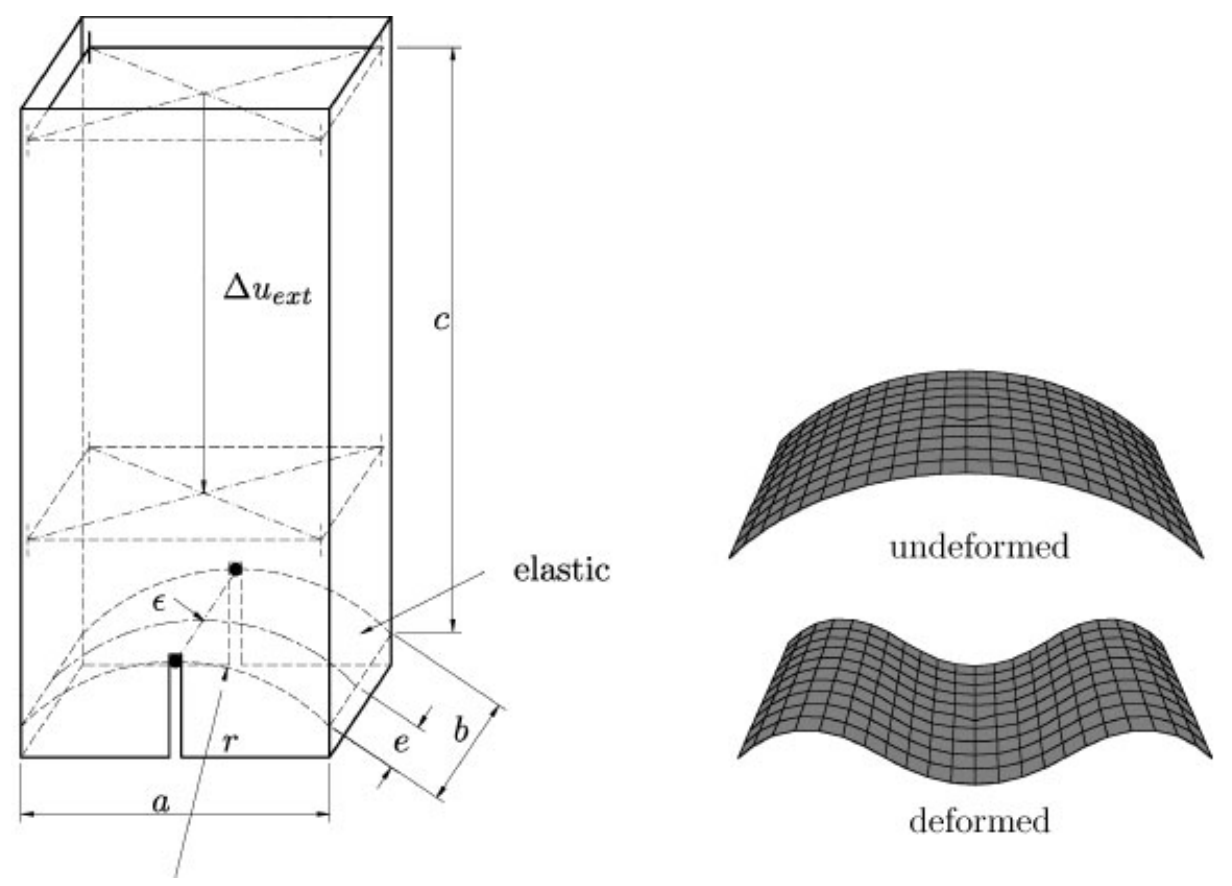

Figure 18. Clamped shell with imperfect geometry under piston loading; $a=10 \mathrm{~cm}$, $b=5 \mathrm{~cm}, c=20 \mathrm{~cm}, r=7 \mathrm{~cm}, e=2.5 \mathrm{~cm}, \Delta u_{\text {ext }}$ controlled piston motion, $\varepsilon$ imperfection; deformed and undeformed shell (right).

the top of the container, see Figure 18. At the bottom shell a small imperfection is introduced to create a unique snap-through behaviour. The two straight sides of the loaded shell are clamped; the centre of the shell contains a small imperfection. Two nodes at both sides of the shell can only slide in vertical direction leading to a somehow symmetric problem. Only the bottom shell is assumed as elastic, the piston and the other walls are assumed as rigid. The solution algorithm is described in Section 8.2. The motion of the piston is controlled directly via the arc-length procedure using an iteration on a normal plane. In Figure 19 the load-displacement curve and the overpressure-displacement curve are shown. It must be noted, however, that the solution could be alternatively performed in two steps by a standard pressure controlled loading again combined with an arc-length procedure. Then after computing the volume change due to the deformation the corresponding piston position can be determined using the gas law.

\subsection{Cylindrical shell with imperfect geometry loaded by vacuum}

The cylinder with imperfect geometry taken from Reference [25] in Figure 20 with a top and a bottom closure is loaded by vacuum which is created by vertical piston motion of the top (elastic modulus $E=2.1 \times 10^{5} \mathrm{~N} / \mathrm{mm}^{2}$, Possion's ratio $v=0.3$, density $\rho=7.85 \times 10^{-6} \mathrm{~kg} / \mathrm{mm}^{3}$ ). In order to achieve rather large deformations the boundary conditions at both ends are almost identical. Both boundary nodes are fixed in in-plane direction, whereas the axial 


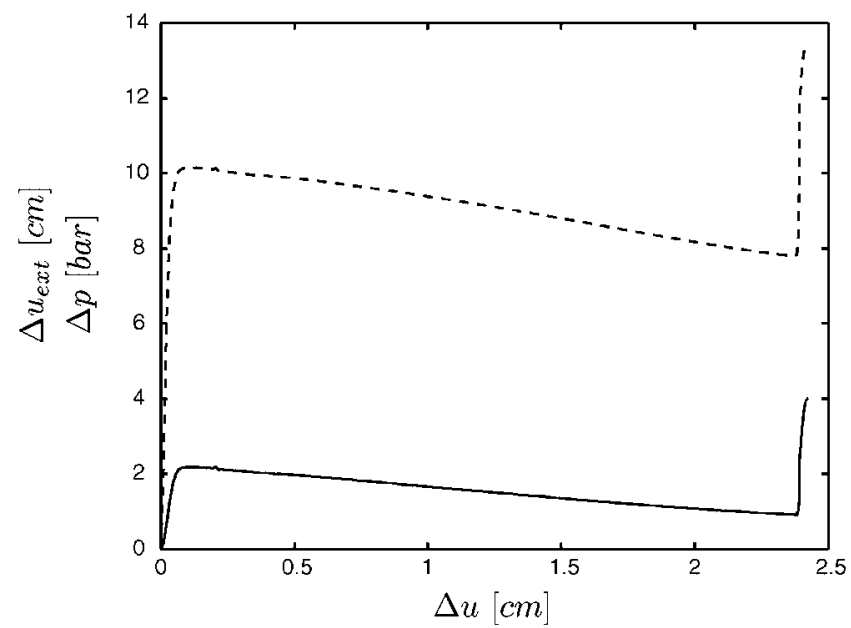

Figure 19. Clamped shell with imperfect geometry under piston loading; --: $\Delta u_{\mathrm{ext}}(\Delta u)$ controlled piston motion-displacement curve, $-: \Delta p(\Delta u)$ overpressure-displacement curve, $\Delta u$ : displacement of point of imperfection.

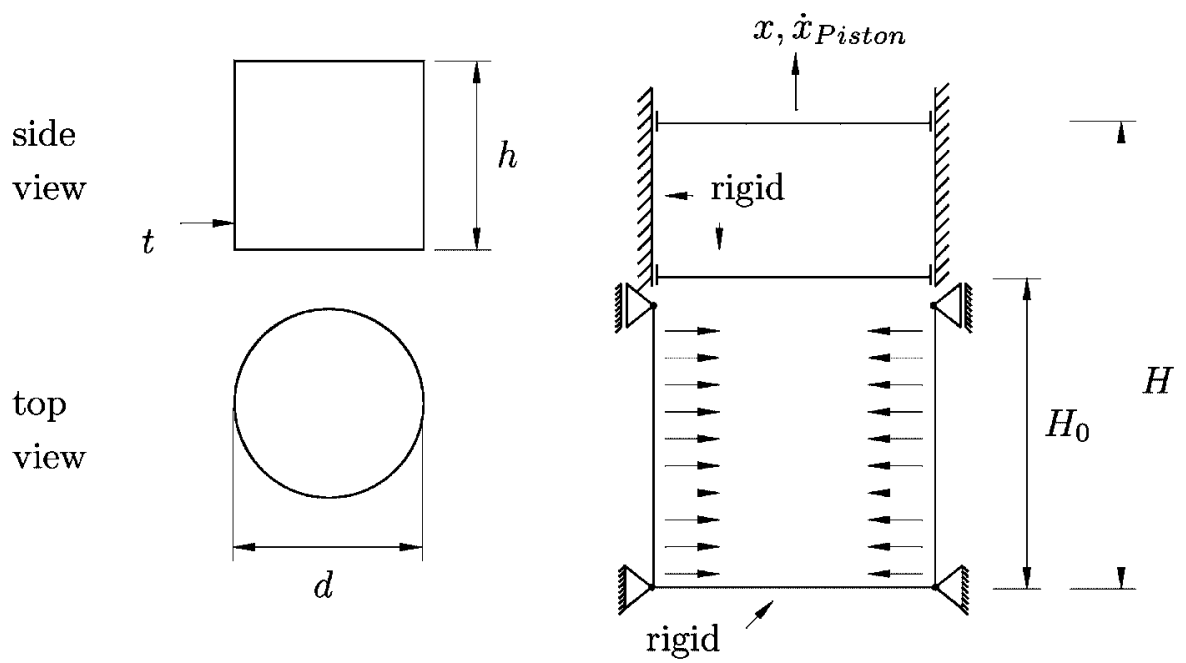

Figure 20. Imperfect cylinder loaded by vacuum created by piston motion of top; loading process; $d=1250 \mathrm{~mm}, h=966 \mathrm{~mm}, t=0.56 \mathrm{~mm}$.

motion-warping - is permitted. Three points at the bottom are fixed in axial direction to provide a non-kinematical system. As the geometry of the cylinder is not perfect the load deformation curve should depict a snap-through type behaviour. This is also computed, however the pressure-motion curve of the top part is almost linear up to the snap-through point, see Figure 21. Further static analyses of the postbuckling path beyond the snap-through point with the piston motion as controlled quantity show serious convergence problems without an 

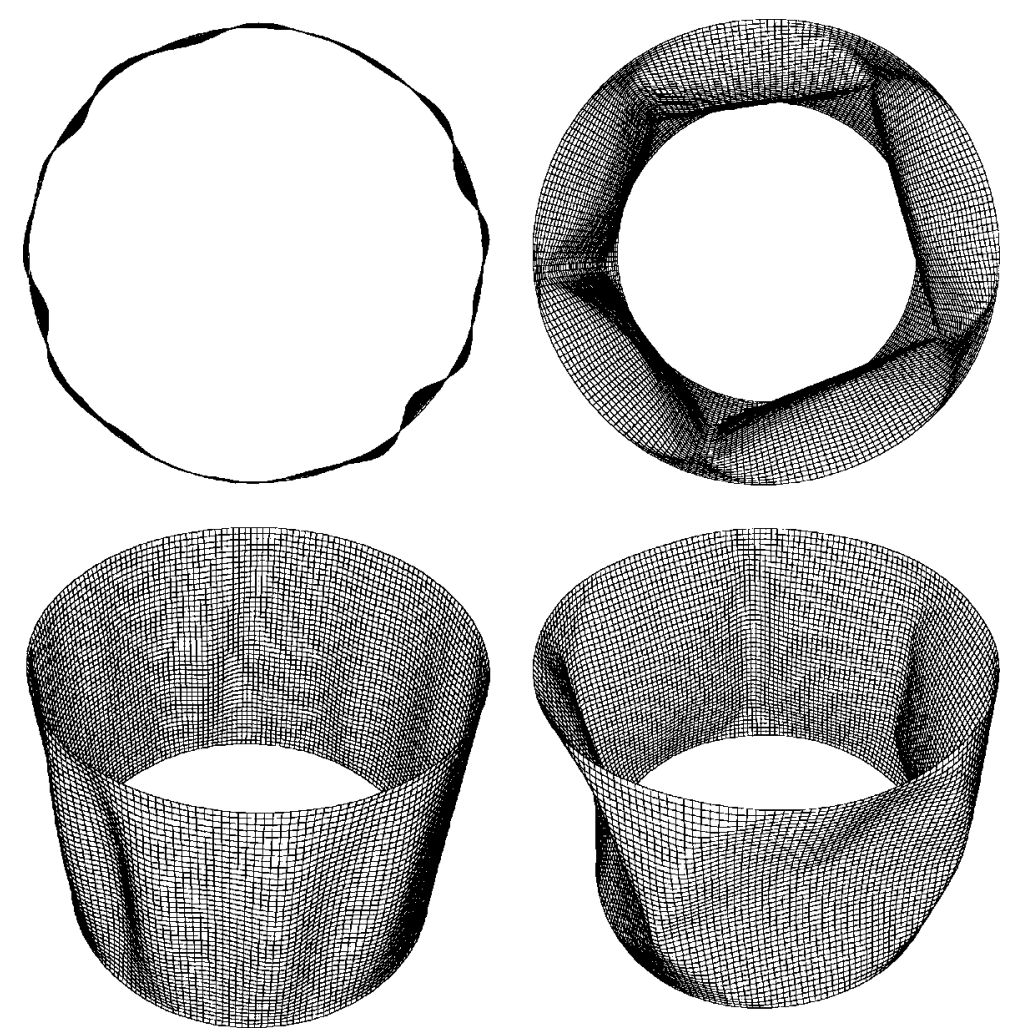

Figure 21. Imperfect cylinder loaded by vacuum created by piston motion of top; side and top view; deformation state at buckling (left); postbuckling state (right).

arc-length scheme. The transient behaviour of the structure is considered-as discussed in References $[25,26]$ for axial loading - leading to a clear pressure deformation path without convergence problems; the corresponding deformed cylinder is shown in Figure 21. Further studies in Reference [27] using LS-DYNA [16] show for an ideal gas with an isentropy constant $\kappa=1.0$ (Boyle's law) that taking the volume change due to wall deformation into account is important for the postbuckling behaviour up to a rather large piston volume. Only for a very large initial piston volume or alternatively the length $H$ the snap-through-behaviour, see Figure 22, changes to a large deformation behaviour with purely increasing pressure-piston-motion curve.

\section{CONCLUSIONS}

The derivations for deformation-dependent loading show that the conservativeness of a structural problem can be proven also when taking the volume of enclosed chambers filled with gas into account by checking the symmetry of the linearized weak form of equilibrium. The particular boundary conditions have been worked out. The discretization with FE leads for each closed chamber to a rank-one update of the stiffness terms. This special structure can be directly used to achieve a very efficient solution scheme, see also Reference [15]. It was 


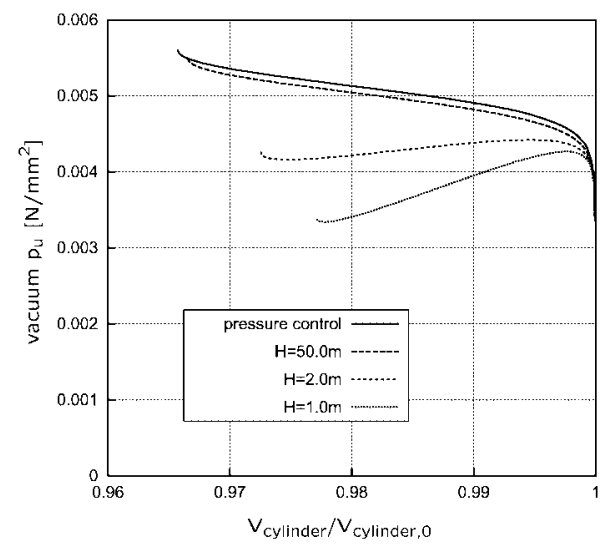

Figure 22. Vacuum vs relative volumetric deformation of the cylinder; variation of initial volume of cylinder and piston.

shown that the consideration of the volume dependence increases the stiffness of a structure including such a closed volume. Within a non-linear analysis and a Newton-type scheme using finite elements the consistent linearization of the deformation-dependent terms leads to quadratic convergence. In combination with arc-length schemes the particular form of the stiffness matrix can be used to achieve a very efficient solution scheme. A very important result of the derivation is that no specific elements are needed to investigate any pneumatic effect.

The numerical examples as e.g. the interaction between two neighbouring chambers under pressure show the necessity of taking the deformation dependence for all terms, in particular, the volume change due to the loading into account. Without these terms correct results cannot be obtained. The stability of the structures increases with the satisfaction of Poisson's law as a further constrained is imposed for each chamber under internal pressure.

\section{REFERENCES}

1. Pearson CE. General theory of elastic stability. Quarterly of Applied Mathematics 1956; 14:133-144.

2. Schweizerhof K. Nonlinear analysis of structures under deformation dependent loading using finite elements. Dissertation, University Stuttgart, 1982 (in German).

3. Schweizerhof K, Ramm E. Displacement dependent pressure loads in nonlinear finite element analyses. Computers and Structures 1984; 18(6):1099-1114.

4. Bufler H. Pressure loaded structures under large deformations. Zeitschrift für Angewandte Mathematik und Mechanik 1984; 64(7):287-295.

5. Schneider H. Fluid loaded membranes undergoing large deformations. Dissertation, University Stuttgart, 1990 (in German).

6. Bufler H. Zur Potentialeigenschaft der von einer Flüssigkeit herrührenden Druckbelastung. Zeitschrift für Angewandte Mathematik und Mechanik 1985; 65(4):130-132.

7. Bufler H. Configuration dependent loading and nonlinear elastomechanics. Zeitschrift für Angewandte Mathematik und Mechanik 1993; 73:4-5.

8. Knebel K. Stability of steel cylinders with unilateral boundary conditions under static and dynamic loading. Dissertation, University Karlsruhe, 1997 (in German).

9. Schweizerhof K, Rumpel T. Volume dependent pressure loading and large deformation finite element analysis of structures. In Trends in Computational Structural Mechanics, Wall WA, Bletzinger K-U, Schweizerhof K (eds). CIMNE: Barcelona, 2001; 386-396. 
10. Rumpel T, Schweizerhof K. Deformation dependent pressure loading at large deformations. GAMM Conference 2001, Zürich (in German).

11. Oden JT, Kubitza WK. Numerical analysis of non-linear pneumatic structures. Proceedings of the First International Colloquium on Pneumatic Structures, IASS, 87-107, Stuttgart, Germany, 1967.

12. Gruttmann F, Tayor RL. Theory and finite element formulation of rubberlike membrane shells using principle stretches. International Journal for Numerical Methods in Engineering 1992; 35:1111-1126.

13. Riches CG, Gosling PD. Numerical analysis of the load-deflection characteristic of pneumatic beam structures. Proceedings of the IASS International Congress '98, Lightweight Structures in Architecture, Engineering and Construction, 1998; 2:883-894.

14. Riches CG, Gosling PD. Pneumatic structures: a review of concepts, applications and analytical methods. Proceedings of the IASS International Congress '98, Lightweight Structures in Architecture, Engineering and Construction 1998; 2:874-882.

15. Bonet J, Wood RD, Mahaney J, Heywood P. Finite element analysis of air supported membrane structures. Computer Methods in Applied Mechanics and Engineering 2000; 190:579-595.

16. Hallquist JO, Tsay CS. LS-DYNA Theoretical Manual. Nonlinear Dynamic Analysis of Structures, vs. 950. Livermore Software Technology Corp.: Livermore, CA, 2000.

17. Sewell MJ. On configuration-dependent loading. Archive for Rational Mechanics and Analysis 1966; 23: 327-351.

18. Romano G. Potential Operators and Conservative Systems. Meccanica 1972; 7:141-146.

19. Zielke G. Inversion of modified symmetric matrices. Journal of the Association for Computing Machinery 1968; 15:402-408.

20. Riks E. An incremental approach to the solution of snapping and buckling problems. International Journal of Solids and Structures 1979; 15:529-551.

21. Ramm E. Strategies for tracing the non-linear response near limit points. In Bathe KJ, Stein E, Wunderlich W (eds). Europe-US-Workshop on Nonlinear Finite Elements Analysis in Structural Mechanics. Springer: Berlin, 1980; 63-89.

22. Crisfield MA. A fast incremental/iterative solution procedure that handels snap-through. Computers and Structures 1981; 13:55-62.

23. Schweizerhof K, Wriggers P. Consistent linearisation for path following methods in non-linear FE analysis. Computer Methods in Applied Mechanics and Engineering 1986; 59:261-279.

24. Hauptmann R, Schweizerhof K. A systematic development of 'solid-shell' element formulations for linear and nonlinear analyses employing only displacement degrees of freedom. International Journal for Numerical Methods in Engineering 1998; 42:49-69.

25. Schweizerhof K, Hauptmann R, Knebel K, Raabe M, Rottner Th. Static and dynamic FE stability analysis of silo shells with nonuniform filling. In Finite Elemente in der Baupraxis, Wriggers P, Meißner U, Stein E, Wunderlich W (eds). Ernst \& Sohn: 1998, pp. 287-306 (in German); Proceedings of the 5th International LS-DYNA Conference, Southfield, Michigan, Livermore Software Technology Corp., Livermore, CA. 1998 (in English).

26. Rottner Th, Schweizerhof K. Transient analysis to compute post-buckling solutions for axially loaded cylindrical shells and sensitivity investigations. Proceedings of the 4th International Colloquium on Computation of Shell and Spatial Structures, IASS-IACM 2000, Chania, Crete, 2000.

27. Metzger A. Collapse analysis of cylindrical containments with time varying gas pressure using the FE method. Diploma Thesis, University Karlsruhe, 2000 (in German). 